\title{
Wireless Injection Locking of Zero-IF Self-Oscillating Mixers
}

\author{
Mabel Pontón ${ }^{\circledR}$, Member, IEEE, Sergio Sancho ${ }^{\circledR}$, Member, IEEE, Amparo Herrera $^{\circledR}$, \\ and Almudena Suárez ${ }^{(1)}$, Fellow, IEEE
}

\begin{abstract}
The recently introduced Zero-IF self-oscillating mixers (SOMs) enable a direct frequency conversion, of interest for the implementation of compact and low consumption radio frequency identification (RFID) tags, among other applications. In previous works, the Zero-IF SOM is placed in only one of the terminals of the wireless link, the other one being based on a conventional scheme. In this article, a system made up of two wirelessly locked Zero-IF SOMs, operating as a frequency upconverter and downconverter, will be analyzed to evaluate its potential for low-cost short-range communications. A complete formulation describing the system under antenna and propagation effects will be presented, which, as a particular case, is able to predict the behavior of the previously proposed Zero-IF SOM, locked by an independent signal. The formulation based on oscillator models extracted from harmonic balance allows deriving design criteria for an optimum and robust performance and can predict the maximum communication range, as well as the stability properties and phase-noise behavior. The operation under modulated conditions is analyzed with a novel envelopetransient formulation, accounting for the time differentiation caused by the propagation effects. The methods have been applied to a system of two Zero-IF SOMs operating at $900 \mathrm{MHz}$.
\end{abstract}

Index Terms-Envelope transient, injection locking, noise analysis.

\section{INTRODUCTION}

$\mathbf{Z}$ ERO-IF self-oscillating mixers (SOMs), proposed in [1] and [2], enable a direct frequency conversion that avoids the need for both an oscillator and a mixer. In comparison with conventional implementations, they reduce the power consumption and size, which is of general interest for compact transmitters and receivers, such as those required for radio frequency identification (RFID) and sensor systems [3]. In the RFID system proposed in [1], the Zero-IF SOM, placed in the tag, is injection locked by the carrier transmitted by the reader.

Manuscript received July 28, 2021; revised October 24, 2021; accepted November 4, 2021. This work was supported in part by the Spanish Ministry of Science and Innovation under Grant PID2020-116569RB-C31 and in part by MCIN/AEI and the European Regional Development Fund (MCIN/AEI/10.13039/501100011033/“ERDF A way of making Europe") under Grant TEC2017-88242-C3-1-R. An earlier version of this paper was presented at the IEEE MTT-S International Microwave Symposium (IMS 2021), Atlanta, GA, USA, June 20-25, 2021 [DOI: 10.1109/IMS19712.2021.9574961]. (Corresponding author: Mabel Pontón.)

The authors are with the Departamento de Ingeniería de Comunicaciones, Universidad de Cantabria, 39005 Santander, Spain (e-mail: mabel.ponton@unican.es; sanchosm@unican.es; herreraa@unican.es; suareza@unican.es).

Color versions of one or more figures in this article are available at https://doi.org/10.1109/TMTT.2021.3127513.

Digital Object Identifier 10.1109/TMTT.2021.3127513
The tag can both demodulate and modulate this carrier, the latter being achieved through the variation of the tag bias voltage. The reader detects this modulation in a conventional fashion, with a local oscillator, mixer, and circulator. As a significant extension of the Zero-IF SOM concept, Burasa et al. [4] proposed a source-less transmitter-receiver system in which multiple Zero-IF SOMs receive and demodulate multiple independent data streams through a direct frequency conversion to baseband. More recently, and considering the increasing demand of multiband wireless systems, the works [5], [6] proposed a concurrent dual-band Zero-IF SOM that, making use of a stepped impedance ring-shape resonator [7], enables a simultaneous compact direct-frequency conversion in two different frequency bands. In addition, Pontón et al. [5], [6] presented an investigation of the stability boundaries of the Zero-IF SOM and its small-signal gain, as well as a strategy to prevent any instantaneous unlocking through a suitable selection of the operation point. Further theoretical studies of the general performance of Zero-IF SOMs have been carried out in [8], which analyzes their main distortion mechanisms and noise behavior.

In view of the potentiality of these novel circuits, this work expands [8] by investigating the possible implementation of a wireless link based on two Zero-IF SOMs, operating as a frequency upconverter and downconverter. A complete formulation describing the system under antenna and propagation effects will be derived, which, as a particular case, is able to predict the behavior of the previously proposed Zero-IF SOM [1], [2], locked by an independent signal. In the most compact implementation, two distinct Zero-IF SOMs are mutually locked through wireless propagation. The Zero-IF SOMs acting as a transmitter are modulated through the variation of a bias voltage. In the Zero-IF SOM acting as a receiver, the modulation gives rise to a time variation of its current consumption, so the signal can be demodulated from the voltage drop in a resistor of the bias circuitry [9]. Here, we will investigate the potentiality of this concept for low-cost and low-consumption short-range communications and derive design criteria for an optimum and robust performance.

The system will be analyzed through a new semianalytical formulation, which relies on oscillator models extracted from harmonic balance (HB) simulations [10], combined with a detailed description of the antenna and propagation effects. Note that the use of numerical models extracted from HB enables a very realistic prediction of the circuit behavior. 
The new formulation, which accounts for the delay in the signal envelope, will allow predicting the maximum communication range, as well as the stability properties and phasenoise behavior. With the aid of this formulation, we will derive the conditions for an optimum operation, considering that the Zero-IF SOM in the receiver must exhibit a high sensitivity, while the one in the transmitter must be able to generate a sufficient oscillation power. Regarding the sensitivity, some previous works demonstrate oscillator designs able to lock to independent input sources with $-100-\mathrm{dBm}$ available power [11], [12]. As will be shown here, the mutually injection-locked operation can be maintained up to distances of several meters with a conventional oscillator design using a single transistor per SOM and low-gain antennas. Note that for this initial proof of concept, two transistor-based SOMs will be considered, though other devices, such as the tunnel diodes recently proposed in [13] and [14], can be more convenient to reduce consumption, size, and cost. We should emphasize that the new formulation is able to predict the behavior of the Zero-IF SOM under unilateral injection locking by an independent carrier (considered in previous works [1], [2]), as a particular case. The operation under modulated conditions will be analyzed with a novel envelope-transient system that accounts for the time differentiation resulting from the propagation effects. The methods have been applied to a system of two Zero-IF SOMs at $900 \mathrm{MHz}$.

This article is organized as follows. Section II describes the wireless link based on two Zero-IF SOMS, as well as the measurement setup that will be used to validate all the new analysis methodologies. Section III presents the calculation of the steady-state solutions. Section IV describes the system behavior versus distance. Section V presents the stability analysis considering the impact of the propagation delay on the signal envelope. Section VI presents the envelope-domain formulation that enables the system analysis in modulated conditions. Finally, Section VII describes the noise analysis.

\section{WIRELESS LINK BASED ON TwO ZERO-IF SOMS}

The wireless link of Fig. 1 is based on two Zero-IF SOMs that make use of a pseudomorphic high-electron-mobility transistor (PHEMT) ATF34143 and are designed as shown in [8]. Though the oscillators are identical, their bias points are different (Fig. 1). They contain two RF ports, which is very convenient for the experimental characterization. In the Zero-IF transmitter, the antenna is connected to the drain port, where the oscillation signal provides the output power $P_{\text {out }}=$ $12 \mathrm{dBm}$; in the Zero-IF receiver, the antenna is connected to the input network where the oscillation signal has a low amplitude and there is a high sensitivity to the received locking signal [15], [16]. In the implementations carried out here, when replacing the matched termination load with the antenna, there is a shift of less than $1 \mathrm{MHz}$ in the oscillator free-running frequency. This shift is relatively small and, as will be shown, poses no problem for the wireless locking of the two oscillators and their capability to transmit/receive modulation signals.

As in any other locked system, the wireless-locked operation can only be maintained within certain parameter ranges. The

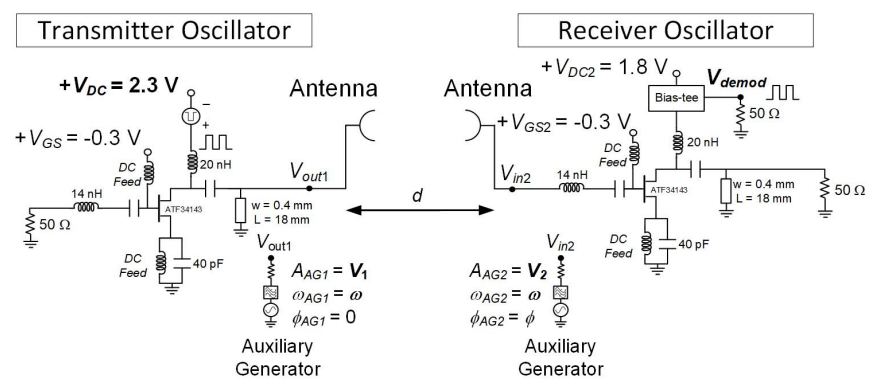

(a)

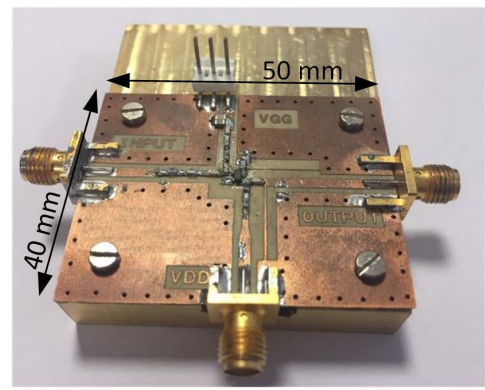

(b)

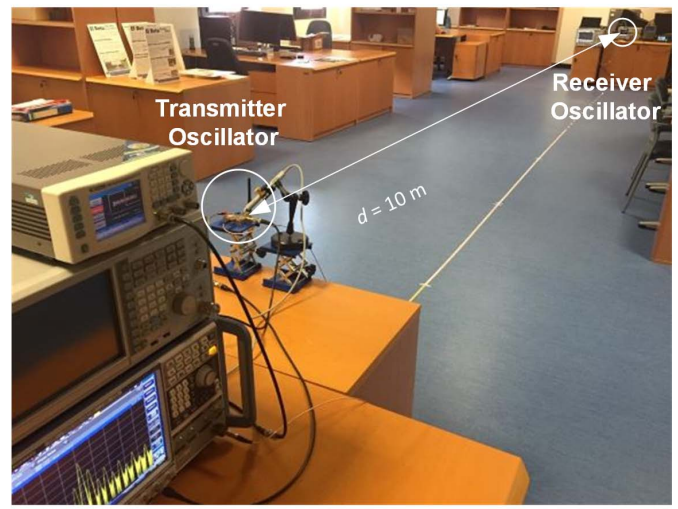

(c)

Fig. 1. Wireless link based on two Zero-IF SOMs. (a) System diagram. The design of two oscillators is the one presented in [8]. They are both based on the PHEMT ATF34143. (b) Photograph of one of the oscillators. (c) Experimental setup for a wireless link at the distance $d=10 \mathrm{~m}$.

system will become unlocked if the free-running frequencies of the two oscillators are too different and/or if the amplitude of the locking signal is too small. Thus, there will be limitations in the excursion of the bias voltage $V_{\mathrm{dc}}$ that affects the free-running frequency of the transmitter oscillator; there will also be limitations in the distance $d$ between the oscillators that affect the amplitude of the locking signal at the input of the receiver oscillator.

Here, for maximum compactness, we will introduce the modulation signal at the drain-bias voltage $V_{\mathrm{DS}}$ of the transmitter oscillator. Besides the frequency modulation inherent to any autonomous system, this will give rise to an amplitude modulation of the transmitted signal, in a manner like the unilateral-locking Zero-IF SOMs in [1]-[4]. Provided that the instantaneous amplitude and frequency do not exceed the locking limits, the receiver oscillator will follow them, so it will also become modulated. Its tracking capability will, of course, be limited by the time constants of the receiver 


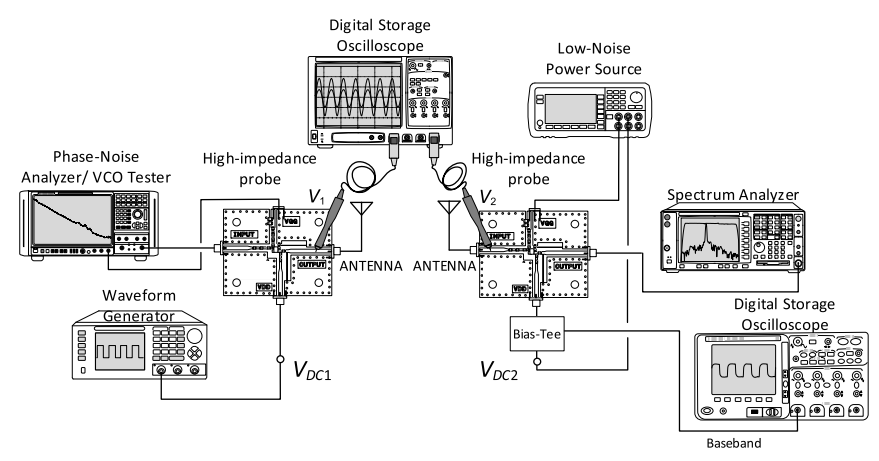

Fig. 2. Experimental setup to characterize the wireless link based on two Zero-IF SOMs. To verify the locked operation, we use an HP E4408B series spectrum analyzer and an R\&S FSWP8 in the spectrum analyzer mode. The modulation signal is obtained with a Tabor WW2572A arbitrary waveform generator. This signal and the demodulated one are displayed in an Agilent DSO91304A digital storage oscilloscope.

Transmitter Oscillator Model
\begin{tabular}{c}
$Y_{1}\left(V_{1}, \omega, V_{D C}\right)=$ \\
$Y_{1 V}\left(V_{1}-V_{o 1}\right)+Y_{1 \omega}\left(\omega-\omega_{o 1}\right)+Y_{D C}\left(V_{D C}-V_{D C o}\right)$ \\
Wireless Channel Model \\
\multicolumn{1}{|c|}{$C(\omega) e^{-j \omega \tau}$} \\
$C(\omega)=\frac{1}{R_{\text {rad }}} \sqrt{G_{1} G_{2}} \frac{c}{\omega d}$ \\
\hline Receiver Oscillator Model \\
\hline$Y_{2}\left(V_{2}, \omega\right)=$ \\
$Y_{2 V}\left(V_{1}-V_{o 1}\right)+Y_{2 \omega}\left(\omega-\omega_{o 2}\right)$ \\
\hline
\end{tabular}

Fig. 3. Complete system description, including the numerical admittancetype models of the two oscillator circuits and the analytical model of the wireless channel.

oscillator, as demonstrated in [8]. The modulation of the receiver oscillator will give rise to a slow time variation of its operation point, so the baseband signal can be extracted from the voltage drop in a resistor of the bias circuitry [1], [2], [9], [1]. The use of a baseband amplifier [4], [17] is also possible.

The measurement setup is shown in Fig. 2. The oscillators are biased using the internal dc supply sources of an R\&S FSWP8 phase-noise analyzer and a Keysight B2962B 6.5 Digit low noise power source. The modulation signal is obtained with a Tabor WW2572A arbitrary waveform generator. The demodulated signal is extracted using a bias-tee connected to the receiver oscillator. Both the modulated and demodulated signals are displayed with an Agilent DSO6034A oscilloscope.

\section{Semianalytical Description}

In this section, we present the system equations; they are based on numerical admittance-type models of the two oscillators, while their wireless interaction is described in an analytical manner, as shown in Fig. 3.

\section{A. Nonlinear System Equations}

In the semianalytical formulation, the oscillators are modeled in terms of their respective admittance functions. It is considered that in standalone operation, each oscillator exhibits at the oscillation frequency a current-to-voltage ratio equal to zero $(Y=0)$ at any node [18]-[23] and, in particular, at the antenna node. Thus, in standalone operation, the two oscillators will fulfill $Y_{1}\left(V_{o 1}, \omega_{o 1}\right)=0$ and $Y_{2}\left(V_{o 2}, \omega_{o 2}\right)=0$ at their respective antenna nodes, where $V_{o i}$ and $\omega_{o i}$, with $i=1$ and 2 , are their free-running voltage amplitudes and frequencies. As discussed, $V_{o 1}$ and $V_{o 2}$ will be significantly different, due to the different location of the antenna. The admittance functions are equal to zero in standalone freerunning operation $\left(Y_{1}=0\right.$ and $\left.Y_{2}=0\right)$, but different from zero under the wireless locked operation, which is due to the presence of the equivalent injection currents. Because of the propagation loss, the wireless injection signals will have a small amplitude, so the admittance of each oscillator can be expressed in a first-order Taylor series expansion about its original free-running solution [19]-[21]. The transmitter oscillator is also linearized with respect to the bias voltage $V_{\mathrm{dc}}$. Note that this voltage will include the modulation signal at a later stage. The linearization with respect to $V_{\mathrm{dc}}$ is not too a strong limitation since the transmitter oscillator must be suitably designed to be as linear as possible versus this bias voltage. Considering a nonlinear behavior with respect to the dc voltage is possible [22] but would prevent reaching insightful analytical expressions. Thus, the oscillators are modeled with the admittance functions

$$
\begin{aligned}
Y_{1}\left(V_{1}, \omega, V_{\mathrm{dc}}\right)= & Y_{1 V}\left(V_{1}-V_{o 1}\right)+Y_{1 \omega}\left(\omega-\omega_{o 1}\right) \\
& +Y_{\mathrm{DC}}\left(V_{\mathrm{dc}}-V_{\mathrm{dc} o}\right) \\
Y_{2}\left(V_{2}, \omega\right)= & Y_{2 V}\left(V_{1}-V_{o 1}\right)+Y_{2 \omega}\left(\omega-\omega_{o 2}\right)
\end{aligned}
$$

where the subscript indicates the variable with respect to which the derivative is calculated, i.e.,

$$
\begin{aligned}
Y_{i V} & \equiv \partial Y_{i}\left(V_{o i}, \omega\right) / \partial V_{i} \\
Y_{i \omega} & \equiv \partial Y_{i}\left(V_{o i}, \omega\right) / \partial \omega_{i} \\
Y_{\mathrm{DC}} & \equiv \partial Y_{1}\left(V_{o 1}, \omega\right) / \partial V_{\mathrm{dc}}
\end{aligned}
$$

and $i=1$ and 2 . The derivatives are just constant complex values extracted from an HB analysis of each oscillator in the standalone operation. They are calculated by introducing an auxiliary generator (AG) into the circuit and applying finite differences to $V_{i}, \omega_{i}$, and $V_{\mathrm{dc}}$, as described in [10] and [23]. The derivatives of the oscillators in Fig. 1 at their corresponding antenna nodes and bias points are shown in Table I. Although not necessary in this work, the admittancetype models of the oscillator circuits could be extracted with these oscillators terminated in the antenna. With this aim, the desired oscillation frequency $\omega_{o}$ is fixed with the AG [10], [18], [22], [23] and one or two circuit element values, together with the AG amplitude, are optimized to fulfill the nonperturbation condition (the ratio between the AG current and voltage equal to zero) at $\omega_{o}$. 
TABLE I

Model Parameter Values

\begin{tabular}{|c|c|}
\hline Transmitter Oscillator & Receiver Oscillator \\
\hline \hline$f_{o 1}=962.58 \mathrm{MHz}, V_{o 1}=2.514 \mathrm{~V}$, & $f_{o 2}=962.58 \mathrm{MHz}$, \\
$V_{D C o}=2.31 \mathrm{~V}$ & $V_{o 2}=0.297 \mathrm{~V}$ \\
\hline$G_{1 V}=-3.09 \times 10^{-2}\left(\Omega^{-1} \cdot V^{-1}\right)$ & $G_{2 V}=-1.98 \times 10^{-1}\left(\Omega^{-1} \cdot V^{-1}\right)$ \\
$B_{1 V}=2.03 \times 10^{-2}\left(\Omega^{-1} \cdot V^{-1}\right)$ & $B_{2 V}=2.90 \times 10^{-1}\left(\Omega^{-1} \cdot V^{-1}\right)$ \\
$G_{1 \omega}=-7.58 \times 10^{-12}\left(\Omega^{-1} \cdot s\right)$ & $G_{2 \omega}=-2.56 \times 10^{-11}\left(\Omega^{-1} \cdot s\right)$ \\
$B_{1 \omega}=2.14 \times 10^{-11}\left(\Omega^{-1} \cdot s\right)$ & $B_{2 \omega}=1.09 \times 10^{-11}\left(\Omega^{-1} \cdot s\right)$ \\
$G_{D C}=-3.05 \times 10^{-2}\left(\Omega^{-1} \cdot V^{-1}\right)$ & \\
$B_{D C}=-2.68 \times 10^{-11}\left(\Omega^{-1} \cdot V^{-1}\right)$ & \\
\hline
\end{tabular}

Assuming a mutually injection-locked operation at the frequency $\omega$, the system is formulated

$$
\begin{aligned}
Y_{1}\left(V_{1}, \omega, V_{\mathrm{dc}}\right) V_{1} e^{j \phi_{o}} & =C(\omega) e^{-j \omega \tau} V_{2} e^{j \phi} \\
Y_{2}\left(V_{2}, \omega\right) V_{2} e^{j \phi} & =C(\omega) e^{-j \omega \tau} V_{1} e^{j \phi_{o}}
\end{aligned}
$$

where $\tau=d / c$ is the time delay, $\phi-\phi_{\mathrm{o}}$ is the phase shift $\left(\phi_{0}\right.$ can be arbitrarily set to zero due to the system autonomy [24]), and $C(\omega)$ is the admittance-dimension factor accounting for the propagation effects [19]. This factor is calculated by combining the expressions of the transmitted power and available power at the receiver [25]. Assuming the same radiation resistance $\left(R_{\mathrm{rad}}=50 \Omega\right)$ in the transmitter and receiver, the factor is

$$
C(\omega)=\frac{1}{R_{\mathrm{rad}}} \sqrt{G_{1} G_{2}} \frac{\lambda}{2 \pi d}=\frac{1}{R_{\mathrm{rad}}} \sqrt{G_{1} G_{2}} \frac{c}{\omega d}=\frac{\eta}{\omega d}
$$

where $G_{1}$ and $G_{2}$ are the antenna gains of the two Zero-IF SOMs, $\lambda$ is the wavelength, and $d$ is the distance. Note that no linearization of $C(\omega)$ is carried out since this function exhibits a strong frequency dependence due to the long delay.

System (1) is nonlinear in $V_{1}$ and $V_{2}$ and can only be solved through a numerical method. In a compact manner, it will be written as

$$
\bar{H}(\bar{v})=\overline{0}
$$

where the vector $\bar{H}$ is composed of the nonlinear functions on the left-hand side of (3), the vector $\bar{v}=\left(V_{1}, V_{2}, \omega, \phi\right)^{T}$ contains the independent variables, and $V_{\mathrm{dc}}$ is the analysis parameter. As in other locked systems [18], [20], [26], under weak coupling conditions, the solutions curves will exhibit turning points (local-global bifurcations [24]), delimiting the locked-operation intervals. Therefore, the resolution of (3) through a monotonic sweep in the analysis parameter is unsuitable. This problem will be overcome here with the arc-length continuation method [27], [28], which makes the vector $\bar{v}$ depend on an additional parameter $s \in \Re$, so system (3) is transformed into

$$
\begin{aligned}
\bar{H}\left(\bar{v}\left(s_{k+1}\right)\right) & =\overline{0} \\
\left|\bar{v}\left(s_{k+1}\right)-\bar{v}\left(s_{k}\right)\right| & =d s
\end{aligned}
$$

where $s_{k}=k d s$ and $d s$ is an increment to be customized. System (6) is solved in steps $k=1, \ldots, n$ following a predictor/corrector procedure, such as the one detailed in [27]. This nonlinear analysis based on arc-length continuation will be applied to predict the circuit response in most of the cases considered in this work. In addition, we will derive an approximate analytical formulation (described in Section III-B), which should provide insight into the system's global behavior.

\section{B. Analytical Study}

For a simplified analytical study, we will neglect in system (1) the second-order terms in the amplitude increments. Splitting the simplified system into real and imaginary parts, one obtains

$$
\begin{aligned}
G_{1 V} \Delta V_{1}+G_{1 \omega} \Delta \omega+G_{\mathrm{DC}} \Delta V_{\mathrm{dc}}= & C_{1}(\omega) \cos (\phi-\omega \tau) \\
B_{1 V} \Delta V_{1}+B_{1 \omega} \Delta \omega+B_{\mathrm{DC}} \Delta V_{\mathrm{dc}}= & C_{1}(\omega) \sin (\phi-\omega \tau) \\
G_{2 V} \Delta V_{2}+G_{2 \omega} \Delta \omega= & C_{2}(\omega) \cos (\phi+\omega \tau) \\
& -G_{2 \omega} \Delta \omega_{o 12} \\
B_{2 V} \Delta V_{2}+B_{2 \omega} \Delta \omega= & -C_{2}(\omega) \sin (\phi+\omega \tau) \\
& -B_{2 \omega} \Delta \omega_{o 12}
\end{aligned}
$$

where

$$
\begin{aligned}
\Delta V_{i} & =V_{i}-V_{o i}, \quad \Delta \omega_{i}=\omega-\omega_{o i} \\
\Delta V_{\mathrm{dc}} & =V_{\mathrm{dc}}-V_{\mathrm{dc} o}, \quad \Delta \omega_{o 12}=\omega_{o 1}-\omega_{o 2} .
\end{aligned}
$$

The following quantities, associated with the signal propagation, have also been defined:

$$
C_{1}(\omega)=C(\omega) \frac{V_{o 2}}{V_{o 1}}, \quad C_{2}(\omega)=C(\omega) \frac{V_{o 1}}{V_{o 2}} .
$$

For a proper operation, one should have a high value of $V_{o 1} / V_{o 2}$, so the coefficient $C_{1}$ will be smaller than $C_{2}$ and the second oscillator will be more sensitive to the locking signal. From the inspection of (4), the constant term $\Delta \omega_{o 12}$ reduces the system sensitivity to the phase shift and thus the locking capabilities, so for an optimum behavior, one should impose $\Delta \omega_{o 12}=0$ or, equivalently, $\omega_{o i}=\omega_{o}$.

We will initially analyze the sensitivity of the locked solution to the bias voltage $V_{\mathrm{dc}}$ in the first oscillator for a fixed distance $d$ between the two Zero-IF SOMs. To address system (7), one must consider that in locked conditions, the phase shift $\phi$ will vary between $0^{\circ}$ and $360^{\circ}$. On the other hand, the system is nonlinear in the common oscillation frequency $\omega$, but linear in $\Delta V_{1}, \Delta V_{2}$, and $\Delta V_{\mathrm{dc}}$. Thus, once $\omega$ is known for each $\phi$, the increments $\Delta V_{1}, \Delta V_{2}$, and $\Delta V_{\mathrm{dc}}$ are directly obtained from (7). In fact, the crucial relationship that enables the prediction of the system behavior is $\omega(\phi)$. To get this, system (7) will be solved for $\Delta \omega$ in terms of the elements after the equal sign, which depends on $\omega$ and $\phi$. This provides the following equation:

$$
H(\omega)=\omega-\omega_{o}+\frac{C_{2}(\omega) \sin \left(\alpha_{2 V}+\omega \tau+\phi\right)}{\left|Y_{2 \omega}\right| \sin \left(\alpha_{2 \omega}-\alpha_{2 V}\right)}=0
$$

where $\alpha_{2 V}$ is the angle of $Y_{2 V}$ and $\alpha_{2 \omega}$ is the angle of $Y_{2 \omega}$. The real equation (10) depends on the characteristics $\left(Y_{2 V}, Y_{2 \omega}\right)$ of the oscillator that is not tuned, $\tau$ and $C_{2}(\omega)$, which increases 
with $V_{o 1} / V_{o 2}$. Equation (10) is easily solved by tracing the zero-value contour $H=0$ in the plane defined by $\phi$ and $\omega$. Next, we will relate $\omega$ with $V_{\mathrm{dc}}$ and with the wireless locking signal. This relationship is easily obtained by solving $\Delta V_{\mathrm{dc}}$ in terms of the already known $(\phi, \omega)$

$$
\begin{aligned}
\omega-\omega_{o}=-\frac{\left|Y_{\mathrm{DC}}\right| \sin \left(\alpha_{\mathrm{DC}}-\alpha_{1 V}\right)}{\left|Y_{1 \omega}\right| \sin \left(\alpha_{1 \omega}-\alpha_{1 V}\right)} \Delta V_{\mathrm{dc}} \\
+C_{1}(\omega) \frac{\sin \left(-\omega \tau+\phi-\alpha_{1 V}\right)}{\left|Y_{1 \omega}\right| \sin \left(\alpha_{1 \omega}-\alpha_{1 V}\right)}
\end{aligned}
$$

where $\alpha_{1 V}, \alpha_{1 \omega}$, and $\alpha_{\mathrm{DC}}$ are the angles of $Y_{1 V}, Y_{1 \omega}$, and $Y_{\mathrm{DC}}$, respectively. From the inspection of (11), in the absence of wireless locking $\left(C_{1}=0\right)$, the frequency will vary linearly with $V_{\mathrm{dc}}$, with a sensitivity solely depending on the characteristics, $Y_{\mathrm{DC}}, Y_{1 V}$, and $Y_{1 \omega}$, of the transmitter oscillator. In the case of the system in Fig. 1, this sensitivity is $40.8 \mathrm{MHz} / \mathrm{V}$. Note that the frequency variation in isolated conditions $\left(C_{1} \neq\right.$ 0 ) would be unbounded, limited only by the validity of the linearization itself. Instead, in (11), the frequency $\omega$ and phase $\phi$ are predetermined by the relationship (10). The second term in (11) also gives rise to the small quasi-sinusoidal variation of $\omega$ versus $\Delta V_{\mathrm{dc}}$.

The solution curve $\omega$ versus $\Delta V_{\mathrm{dc}}$ for the bilateral system in Fig. 1, with $V_{o 1}=2.52 \mathrm{~V}$ and $V_{o 2}=0.29 \mathrm{~V}$, is shown in Fig. 4(a). Two different values of the antenna gain have been assumed: $G=2 \mathrm{~dB}$ and $G=6 \mathrm{~dB}$. For the lower gain $(G=2 \mathrm{~dB})$, we have considered the distances $d=5 \mathrm{~m}$ and $d=10 \mathrm{~m}$. For the higher gain $(G=6 \mathrm{~dB})$, the distance is $d=20 \mathrm{~m}$. In all cases, the solution curve is closed (see expanded view) but nearly degenerated into the straight line that would be obtained with (11) for $C_{1}=0$. Its form of variation will be seen in more detail in Section III. In fact, for each pair $G$ and $d$, the locked operation is maintained between the two turning points of the closed curve, which corresponds to local/global bifurcations [24] from which the two oscillators get unlocked. The antenna gain $G$ and the distance $d$ affect the excursion of the oscillation frequency, which is larger for higher $G$ and smaller $d$. The curves exhibit a hardly noticeable undulation due to the sinusoidal dependences of (12) and (13); this will be more evident in the analysis of Section III. Note that only one section of each closed curve in Fig. 4(a) (between the two turning points) is stable, as demonstrated in Section IV.

For each pair of $G$ and $d$ values, results obtained through the numerical resolution of the nonlinear system (5) are also over the same axis and their boundaries are indicated with circles. In comparison, the simplified analysis based on (5) and (10) tends to overestimate the boundaries. Experimental points obtained with the R\&S FSWP8 in the spectrum analyzer mode (Fig. 2) for $G=2 \mathrm{~dB}$ at the two distances $d=5$ and $10 \mathrm{~m}$ are superimposed. Note that due to the lab space limitations, it was not possible to perform measurements for $d=20 \mathrm{~m}$.

\section{Particularization to the Unilateral Case}

At this point, it might be interesting to compare the relationship $\omega\left(V_{\mathrm{dc}}\right)$ provided by (5) and (10) with the much simpler

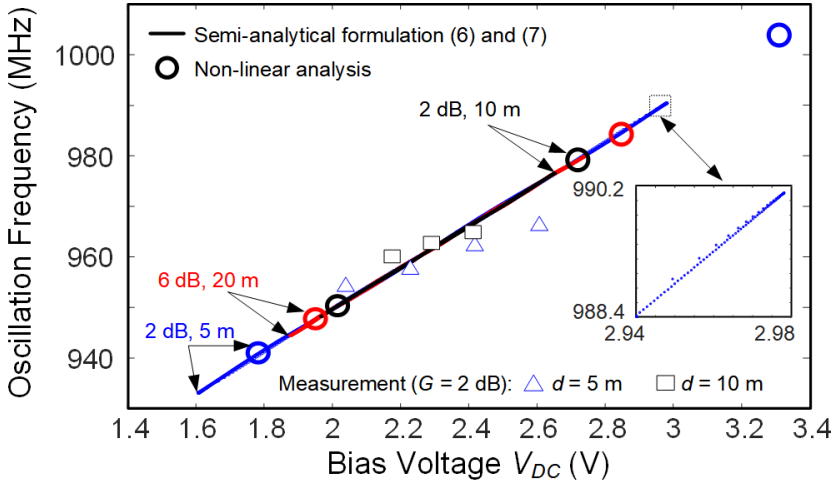

(a)

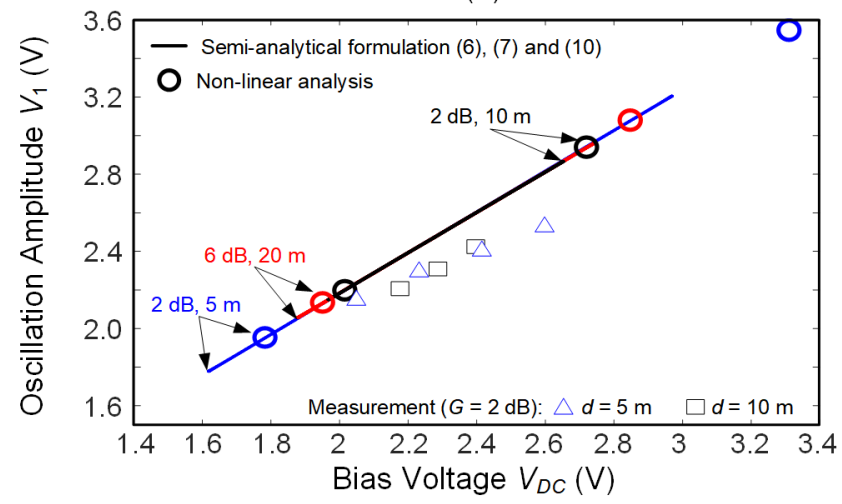

(b)

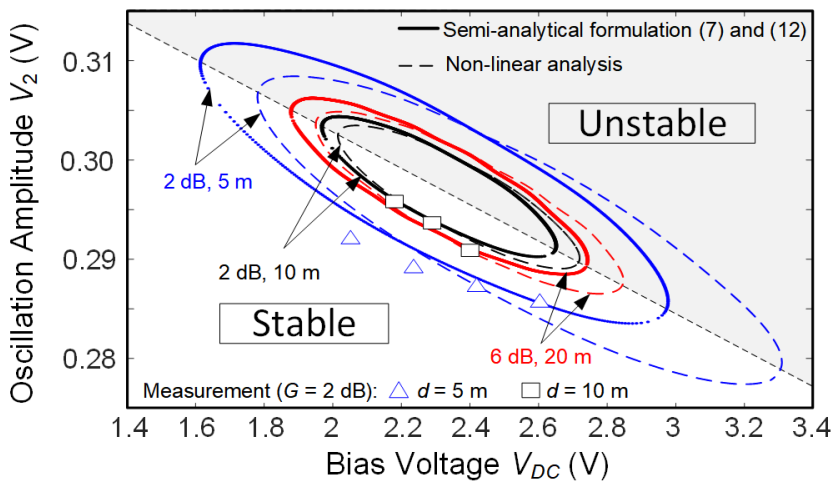

(c)

Fig. 4. Mutually locked solution of the system in Fig. 1 versus the bias voltage $V_{\mathrm{dc}}$ in the transmitter oscillator under the antenna gain values $G=$ $2 \mathrm{~dB}$ and $G=6 \mathrm{~dB}$. For the lower gain $(G=2 \mathrm{~dB})$, the distances $d=5 \mathrm{~m}$ and $d=10 \mathrm{~m}$ are considered. For the higher gain $(G=6 \mathrm{~dB})$, the distance is $d=20 \mathrm{~m}$. The results of the nonlinear formulation and the simplified one are compared. Measurement points are superimposed for $G=2 \mathrm{~dB}$ and the two distances $d=5$ and $10 \mathrm{~m}$. (a) Oscillation frequency. (b) Amplitude variation in the transmitter oscillator. (c) Amplitude variation in the receiver oscillator.

one that would be obtained in the unilateral case, governed by the two last equations in (7) only. In that case, $\omega$ would agree with the independent frequency of the transmitter $\omega_{\text {in }}$, and the system equations would be

$$
\begin{aligned}
G_{2 V} \Delta V_{2}+G_{2 \omega}\left(\omega_{\text {in }}-\omega_{o}\right) & =C_{2}\left(\omega_{\text {in }}\right) \cos \left(\phi+\frac{\omega_{\text {in }} d}{c}\right) \\
B_{2 V} \Delta V_{2}+B_{2 \omega}\left(\omega_{\text {in }}-\omega_{o}\right) & =-C_{2}\left(\omega_{\text {in }}\right) \sin \left(\phi+\frac{\omega_{\text {in }} d}{c}\right) .
\end{aligned}
$$

Initially, the above system should be solved for $\omega_{\text {in }}-\omega_{o}$ in terms of $\phi+\omega_{\text {in }} d / c$. For simplicity, we will assume that 
the independent frequency is varied as $\omega_{\text {in }}=\omega_{o}+K_{\omega} \Delta V_{\mathrm{dc}}$, where $K_{\omega}$ is the frequency sensitivity. Replacing this expression into (12), one obtains

$$
\begin{aligned}
& K_{\omega} \Delta V_{\mathrm{dc}} \\
& =-\frac{C_{2}\left(\omega_{o}+K_{\omega} \Delta V_{\mathrm{dc}}\right) \sin \left(\alpha_{2 V}+\omega_{o} \tau+K_{\omega} \Delta V_{\mathrm{dc}} \tau+\phi\right)}{\left|Y_{2 \omega}\right| \sin \left(\alpha_{2 \omega}-\alpha_{2 V}\right)} .
\end{aligned}
$$

Note that there would only be locked solutions in the $\Delta V_{\mathrm{dc}}$ interval fulfilling the above relationship, and the corresponding frequencies would be calculated by replacing $\Delta V_{\mathrm{dc}}$ in $\omega_{\text {in }}=\omega_{o}+K_{\omega} \Delta V_{\mathrm{dc}}$. In practice, the independent signal at $\omega_{\text {in }}$ would be generated through a synthesizer in most cases and the control parameter would be the frequency-division order. The complete scheme would be a conventional one, instead of the compact and low-cost solution proposed here.

\section{Oscillation Amplitudes}

In the case of the two mutually injection-locked Zero-IF SOMs, the oscillation amplitudes are obtained by solving $\Delta V_{1}$ and $\Delta V_{2}$ from (7) in terms of the already known $(\phi, \omega)$. These can be composed with $\Delta V_{\mathrm{dc}}(\phi, \omega)$ to obtain their variation versus $V_{\mathrm{dc}}$. Fig. 4(b) presents the variation of $V_{1}$. Results of the nonlinear formulation solved through arc-length continuation are also shown. For each pair $G$ and $d$ of gain and distance values, the amplitude $V_{1}$ describes a closed curve nearly degenerated into a straight line, as in the case of the oscillation frequency $\omega$. The simplified formulation overestimates the boundaries of these closed curves, which agrees with those of $\omega$. For a small $C_{1}$, the location and slope of the straight line (into which the closed curve nearly degenerates) are the same as those obtained in uncoupled conditions $\left(C_{1}=0\right)$

$$
\Delta V_{1}=\frac{-\left|Y_{\mathrm{DC}}\right| \sin \left(\alpha_{1 \omega}-\alpha_{V \mathrm{dc}}\right)}{\left|Y_{1 v}\right| \sin \left(\alpha_{1 \omega}-\alpha_{1 V}\right)} \Delta V_{\mathrm{dc}}
$$

However, as concluded in Section III-B, in these uncoupled conditions, the variation of $\Delta V_{1}$ would be unbounded.

The amplitude increment in the second oscillator is

$$
\Delta V_{2}=V_{2}-V_{o 2}=\frac{C_{2}(\omega) \sin \left(\omega \tau+\phi+\alpha_{2 \omega}\right)}{\left|Y_{2 V}\right| \sin \left(\alpha_{2 \omega}-\alpha_{2 V}\right)}
$$

It is relevant to note that the above expression is formally identical to the one that would be obtained under unilateral locking using the system (12). However, the frequency $\omega$ in (15) is not independent, but the one resulting from (10). One obtains the closed curves in Fig. 4(c), each for a different pair of $G$ and $d$ values. As gathered from Fig. 4(c), the second oscillator (without a tuning voltage) is more sensitive to the locking signal than the first one, so the closed solution curves are wider. The simplified and nonlinear formulations exhibit stronger discrepancies for a higher $G$ and a smaller $d$.

In the experimental characterization, the voltages are obtained using Agilent 1169 differential probes (Fig. 2), which allows testing differential and single-ended signals up to $12 \mathrm{GHz}$, and an Agilent DSO91304A Digital Storage Oscilloscope. Measurement points for $G=2 \mathrm{~dB}$ and the two distances $d=5 \mathrm{~m}$ and $10 \mathrm{~m}$ are superimposed in Fig. 4(b) and (c). In the two cases, the stable section is the lower one, in agreement with the results of the stability analysis to be presented in Section III.

To summarize the results obtained so far, system (4) accounts for bilateral injection locking through wireless propagation. For small $C_{1}$, the behavior approaches the one obtained under unilateral locking. Under $C_{1}=0$, the same new formulation can be used to analyze the behavior of a single Zero-IF SOM injected by a locking signal undergoing propagation effects.

\section{Analysis Versus Distance}

In this section, we will perform an analysis of the mutually locked system versus the distance $d$ between the two Zero-IF SOMs. This analysis, of paramount importance for the wireless link, provides the maximum distance up to which the locked operation can be maintained. As a particular case, we will also consider the case of a single Zero-IF SOM under unilateral locking.

\section{A. Frequency and Amplitude Variation}

At a constant bias voltage $V_{\mathrm{dc}}$, the system equations (under the first-order amplitude approximation) are given by

$$
\left.\begin{array}{l}
G_{1 V} \Delta V_{1}+G_{1 \omega} \Delta \omega=C_{1}(\omega) \cos (\phi-\beta)-G_{\mathrm{DC}} \Delta V_{\mathrm{dc}} \\
B_{1 V} \Delta V_{1}+B_{1 \omega} \Delta \omega=C_{1}(\omega) \sin (\phi-\beta)-B_{\mathrm{DC}} \Delta V_{\mathrm{dc}}
\end{array}\right\}
$$

where $C_{1}$ and $C_{2}$ depend on $d$ as shown in (4) and (9) and $\beta=\omega d / c$. Again, the crucial variable of system (16) is $\omega$. Once this variable is known, the rest can be obtained in a simple manner, as shown later in this section. For simplicity, we will particularize the following derivation to the case $\Delta V_{\mathrm{dc}}=0$. To obtain $\omega(d)$, we will express the system (16) in terms of $\cos (\phi)$ and $\sin (\phi)$, which together with $\Delta V_{1}$ and $\Delta V_{2}$ will be the system unknowns. Considering that $\cos ^{2} \phi+\sin ^{2} \phi=1$, one obtains (for $\Delta V_{\mathrm{dc}}=0$ ) the following single scalar equation:

$$
\begin{aligned}
& H(\omega) \\
& \quad=\left[C_{2}^{2} T_{1}^{2}+C_{1}^{2} T_{2}^{2}+2 C_{1} T_{1} C_{2} T_{2} \cos \left(\alpha_{V 2}-\alpha_{V 1}\right)\right] \Delta \omega^{2} \\
& \quad=C_{1}^{2} C_{2}^{2} \sin ^{2}\left(\alpha_{V 2}+\alpha_{V 1}+2 \beta\right)=0
\end{aligned}
$$

where the following definitions have been introduced:

$$
\begin{aligned}
& T_{1}=\left|Y_{1 \omega}\right| \sin \left(\alpha_{1 \omega}-\alpha_{1 V}\right) \\
& T_{2}=\left|Y_{2 \omega}\right| \sin \left(\alpha_{2 \omega}-\alpha_{2 V}\right) .
\end{aligned}
$$

Note that $C_{1}, C_{2}$, and $\beta$ depend on $d$. Equation (17) provides a contour in the plane defined by $d$ and $\omega$, which is composed of two disconnected curves. These two curves, in the case of the system in Fig. 1, are shown in Fig. 5(a), where the distance has been varied in the interval 4.85-5.15 $\mathrm{m}$. Two values of antenna gain, $G=2 \mathrm{~dB}$ and $G=6 \mathrm{~dB}$, have been considered to better evidence the oscillatory behavior about the free-running frequency $\omega_{o}$. Note that the frequency variations versus the distance $d$ at a constant $V_{\mathrm{dc}}$ in the first oscillator, shown in Fig. 5(a), are very small [compare with Fig. 4(a)], which favors the system robustness. Note that this analysis is 


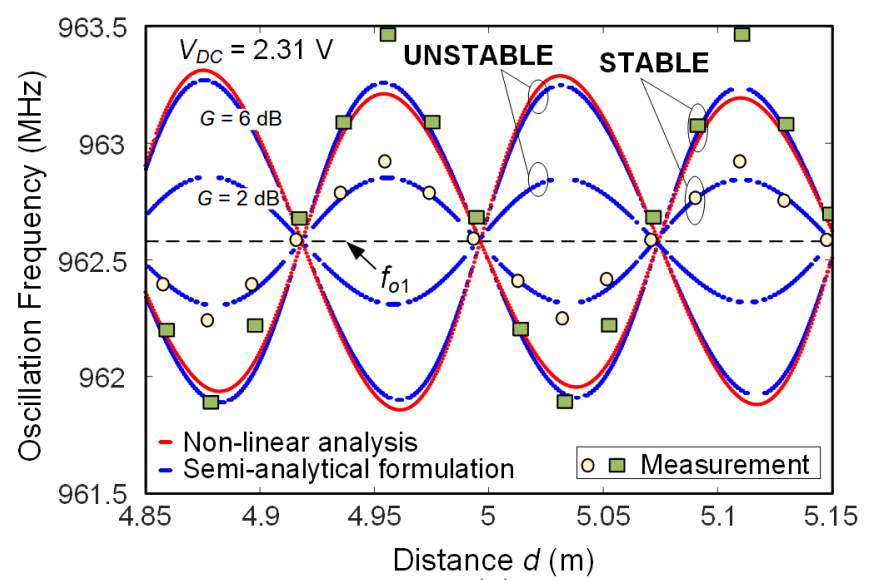

(a)

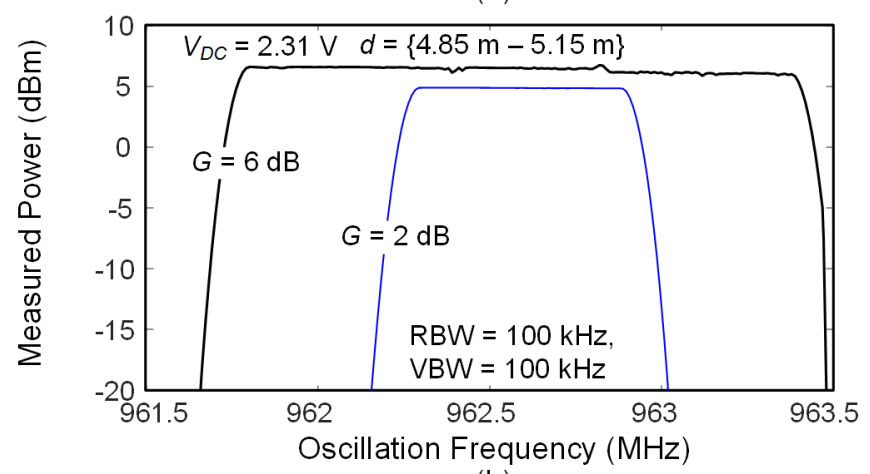

(b)

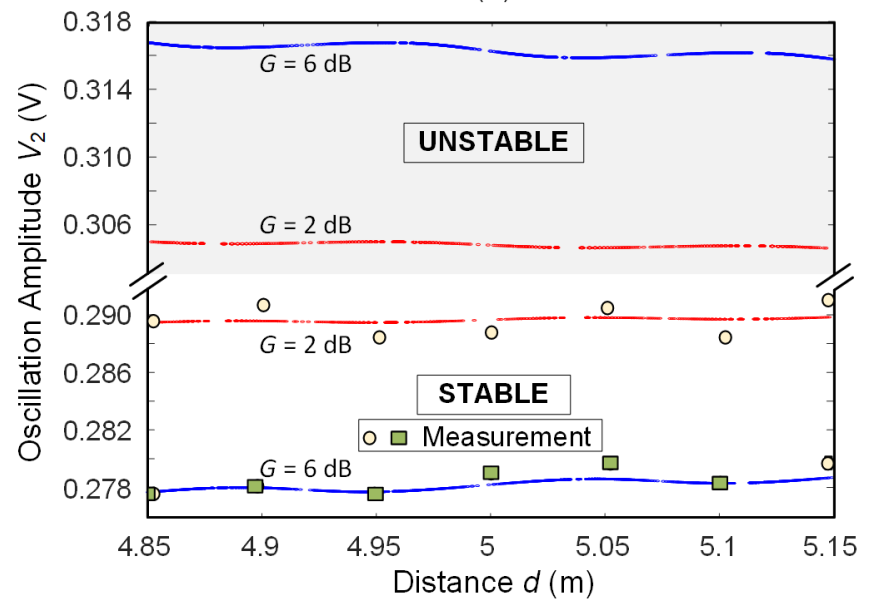

(c)

Fig. 5. Variation of the locked-system solutions versus the distance $d$ between the two oscillators for a constant bias voltage $\Delta V_{\mathrm{dc}}=0 \mathrm{~V}$. There are two coexistent solution curves. (a) Frequency variation in the interval $4.85-5.15 \mathrm{~m}$. Measurement points (corresponding to the stable solution) are superimposed. (b) Experimental measurements obtained using the max hold mode in the spectrum analyzer and continuously varying the distance between the antennas in a relatively small range about $5 \mathrm{~m}$. (c) Variation of the second oscillator amplitude $V_{2}$.

performed under a constant value of $V_{\mathrm{dc}}\left(\Delta V_{\mathrm{dc}}=0\right)$, so the frequency variations are solely generated by mutual locking effects and will not exist in the case of unilateral locking $\left(C_{1}=0\right)$ treated in Section IV-B. For each $d$, the two solutions in Fig. 5(a) would correspond to those obtained through the analysis in Section II at $\Delta V_{\mathrm{dc}}=0$, that is, they are different

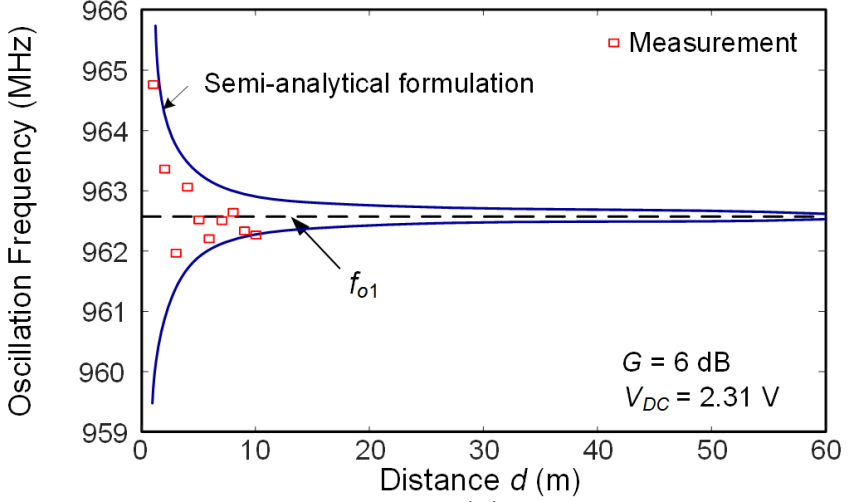

(a)

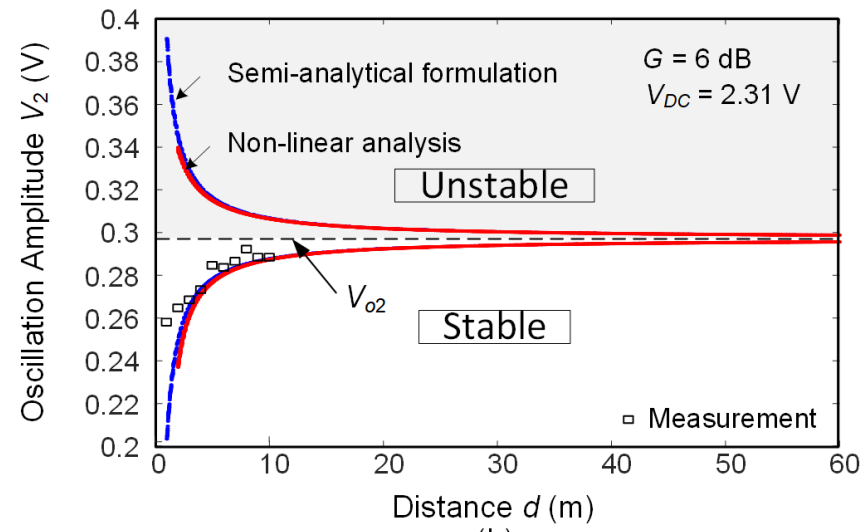

(b)

Fig. 6. Variation of the locked-system solutions in a large distance interval. (a) Envelopes of the maxima and minima of the oscillation frequency curves in Fig. 5(a) up to $d=60 \mathrm{~m}$. (b) Variation of the second-oscillator amplitude $V_{2}$.

projections of the same solution surface in the space $\omega\left(V_{\mathrm{dc}}, d\right)$. As expected, the frequency excursion is larger for a higher gain [Fig. 5(a)]. One of the curves is Fig. 5(a) stable (with measurement points superimposed) and the other unstable, as will be shown in Section III.

To understand the quasi-sinusoidal variation, one can detect the distance points at which frequency curves take a value equal to the free-running one $\omega_{0} . \Delta \omega=0$ in (17) has on at these distance points and the following condition is fulfilled:

$$
\sin \left(\alpha_{V 2}+\alpha_{V 1}+2 \beta\right)=0 .
$$

Because $\beta=\omega d / c$, the distance values at which $\Delta \omega=0$ are spaced in $\lambda / 2$. Fig. 5(b) shows the experimental measurements obtained using the max hold mode in the spectrum analyzer (Fig. 2) and continuously varying the distance $d$ between the antennas in the interval $4.85-5.15 \mathrm{~m}$. This measurement has been carried out for two values of antenna gain: $G=2 \mathrm{~dB}$ and $G=6 \mathrm{~dB}$, and as predicted in Fig. 5(a), a larger frequency excursion is obtained for a higher antenna gain.

Next, we will carry out an analysis in a larger distance interval, up to $d=60 \mathrm{~m}$. To better evidence the frequency variations, in Fig. 6(a), we have represented the envelopes of maxima and minima of the frequency curves, which are, in fact, the extensions of those in Fig. 5(a) when increasing $d$. Note that the maximum locking distance under $\Delta V_{\mathrm{dc}}=0$ is theoretically infinite as shown in Section IV-B, and the 
asymptote is $\omega=\omega_{0}$. Even at $d=60 \mathrm{~m}$, the locked operation has a nonnegligible effect on the oscillation frequency, which is an indication of a quite robust behavior. Measurements up to $d=10 \mathrm{~m}$ have been superimposed.

Once the frequency $\omega$ is known, one can directly solve (16) (expressed as a linear system in terms of $\Delta V_{1}, \Delta V_{2}, \cos \phi$, and $\sin \phi$ ) to obtain the two amplitudes $V_{1}$ and $V_{2}$. The voltage $V_{1}$ in the transmitter oscillator is hardly affected by the distance $d$ since it mostly obeys the variation of its bias voltage $V_{\mathrm{dc}}$. On the other hand, the voltage $V_{2}$ in the receiver oscillator varies as shown in Figs. 5(c) and 6(b), which consider short- and long-distance intervals, respectively. The two figures show the variation of the two solutions obtained in Fig. 4(c) for $\Delta V_{\mathrm{dc}}=0$ versus the distance $d$. Measurements corresponding to the stable curve are superimposed in the two cases. As expected, the amplitude excursion is larger for a higher gain and so is the quasi-sinusoidal variation [Fig. 5(c)].

\section{B. Particularization to the Unilateral Case}

We can also particularize the analysis versus the distance $d$ to the simpler case in which the second oscillator is locked by a wirelessly transmitted independent signal at the frequency $\omega_{\text {in }}$. Squaring the two real equations in (12), adding the squared terms on the left-hand side, and solving for $\Delta V_{2}$, one obtains the expression

$$
\begin{aligned}
& \Delta V_{2}=- \frac{\left|Y_{2 \omega}\right| \cos \left(\alpha_{2 \omega}-\alpha_{2 V}\right) \Delta \omega_{\text {in }}}{\left|Y_{2 V}\right|} \\
& \pm \frac{\sqrt{\begin{array}{l}
\left(2\left|Y_{2 V}\right|\left|Y_{2 \omega}\right| \cos \left(\alpha_{2 \omega}-\alpha_{2 V}\right) \Delta \omega_{\text {in }}\right)^{2} \\
-4\left|Y_{2 V}\right|^{2}\left(\left|Y_{2 \omega}\right|^{2} \Delta \omega_{\text {in }}^{2}-\left(\frac{V_{o 1}}{V_{o 2}}\right)^{2} \frac{\eta^{2}}{\omega_{\text {in }}^{2} d^{2}}\right)
\end{array}}}{2\left|Y_{2 V}\right|^{2}} .
\end{aligned}
$$

As can be gathered, there are two solutions for each $\omega_{\text {in }}$, provided that this frequency is inside the locking bandwidth (as required for a positive radicand). Unlike (20), in the bilateral case, the locked-oscillation frequency changes with the distance $d$, as shown in Figs. 5(a) and 6(a). Despite this, the explicit equation (20) explains a feature that is common to the two systems: the existence of a maximum distance $d_{\max }$ up to which the locking can be maintained. Representing the input frequency as $\omega_{\mathrm{in}}=\omega_{o}+K_{\omega} \Delta V_{\mathrm{dc}}$ and making the radicand of (18) equal to zero, for each $\Delta V_{\mathrm{dc}}$, the distance $d_{\max }$ is given by

$$
\begin{aligned}
& d_{\max }=\frac{V_{o 1}}{V_{o 2}} \\
& \times \frac{c \sqrt{G_{1} G_{2}}}{\left|Y_{2 \omega}\right|\left(\omega_{o}+K_{\omega} \Delta V_{\mathrm{dc}}\right) R_{\mathrm{rad}} K_{\omega}\left|\Delta V_{\mathrm{dc}}\right| \sin \left(\alpha_{2 \omega}-\alpha_{2 V}\right)} .
\end{aligned}
$$

The distance $d_{\max }$ ideally tends to infinite for $\Delta V_{\mathrm{dc}}=0$, which explains the two asymptotic curves in Fig. 6(b) and (c). For $\Delta V_{\mathrm{dc}} \neq 0$, it increases with the antenna gain and decreases, as expected, with $\left|Y_{2 \omega}\right|$ and, thus, with quality factor of the locked oscillator.

\section{Maximum Locking Distance in the Bilateral Case}

As already described, the solution curve $V_{2}(d)$ in the more complex bilateral case is obtained by first calculating $\omega(d)$

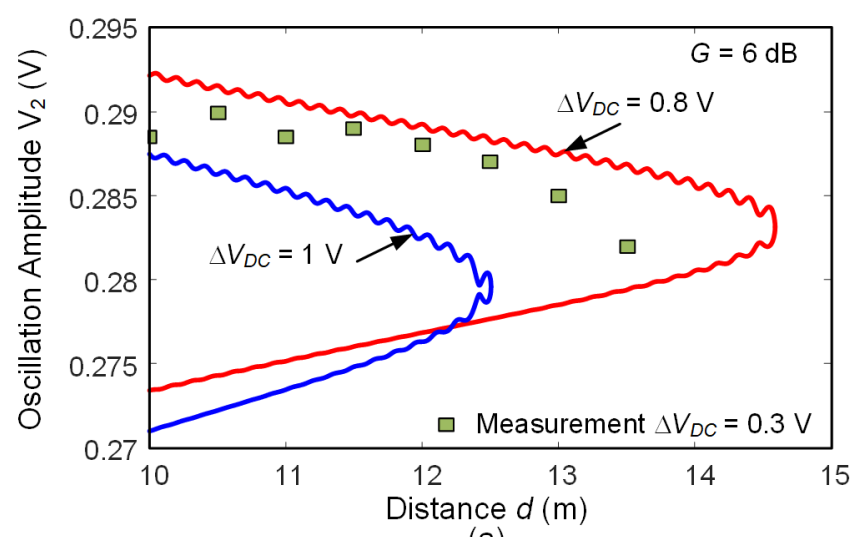

(a)

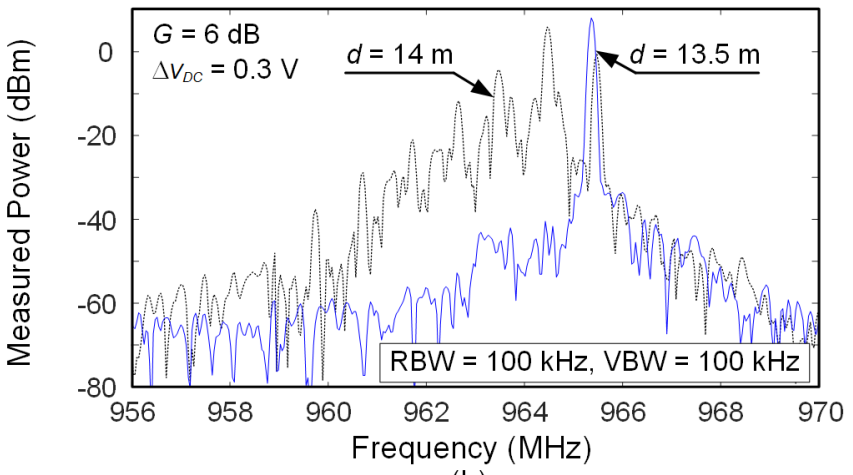

(b)

Fig. 7. Variation of the second-oscillator amplitude $V_{2}$ versus the distance $d$. (a) Solution curves for two different values of the bias voltage: $\Delta V_{\mathrm{dc}}=$ $1 \mathrm{~V}$ and $\Delta V_{\mathrm{dc}}=0.8 \mathrm{~V}$. Measurements are superimposed for the second case. The turning point delimits the locking interval. (b) Spectra measured at $d=$ $14 \mathrm{~m}$, in unlocked conditions, and just after locking $(d=13.5 \mathrm{~m})$.

from (17) and then solving for $\Delta V_{2}$ from (16). Fig. 7(a) presents the variation of $V_{2}$ versus the distance $d$ in the mutually coupled system for two values of $\Delta V_{\mathrm{dc}}$ different from zero. The curves have been obtained by solving system (3) numerically through arc-length continuation. In a manner similar to the unilateral case described through (20), the maximum distance, $d_{\max }$, up to which the system keeps locked decreases for a larger $\left|\Delta V_{\mathrm{dc}}\right|$. At $d_{\max }$, the curves exhibit a turning point, which corresponds to a local/global bifurcation [24] from which the two oscillators get unlocked. As in previous analyses, a small quasi-sinusoidal variation is observed along each solution curve, which is due to the sinusoidal terms in (18) resulting from the delay $\tau$. For $\Delta V_{\mathrm{dc}}$ considered in the analysis, there were discrepancies with the measurement results, mostly attributed to inaccuracies in the model of the transistor device; in standalone operation, the experimental output power exhibited a higher slope versus $V_{\mathrm{dc}}$ than the simulation results. Thus, we had to reduce $\Delta V_{\mathrm{dc}}$ in the measurements. The experimental points obtained for $\Delta V_{\mathrm{dc}}=0.3 \mathrm{~V}$ have been added in Fig. 7(a). The spectra measured in unlocked conditions and just after locking are shown in Fig. 7(b). At $d=14 \mathrm{~m}$, the spectrum is quasiperiodic with a small beat frequency and the typical triangular shape [15], [16]. At $d=13.5 \mathrm{~m}$, very close to the boundary of the locking range, the spectrum is periodic. 
Because two distinct solution curves have been detected, a stability analysis to distinguish the physical solutions will be mandatory. This will be the object of Section V.

\section{Stability AnAlysis}

This section presents a stability analysis of the two mutually locked oscillators that account for the effect of the propagation delay on the signal envelope. Note that, for higher accuracy, this stability analysis will be applied to the nonlinear system (1), instead of the first-order approximation. To facilitate the derivations, system (1), providing the steady-state solution $\left(V_{1}, V_{2}, \phi, \omega\right)$, will be rewritten as

$$
\begin{aligned}
& H_{1}\left(X_{1}, X_{2}, \omega\right) \equiv Y_{1}\left(V_{1}, \omega\right) X_{1}+Y_{c} X_{2}=0 \\
& H_{2}\left(X_{1}, X_{2}, \omega\right) \equiv Y_{2}\left(V_{2}, \omega\right) X_{2}+Y_{c} X_{1}=0
\end{aligned}
$$

where $X_{i}=V_{i} e^{j \phi_{i}}$ with $\phi_{1}=0, \phi_{2}=\phi$, and

$$
\begin{aligned}
& Y_{i}=Y_{i V}\left(V_{i}-V_{o i}\right)+Y_{i \omega}\left(\omega-\omega_{o i}\right)+\delta_{i}^{1} Y_{\mathrm{DC}}\left(V_{\mathrm{dc}}-V_{\mathrm{dc} o}\right) \\
& Y_{c}=-C(\omega) e^{-j \omega \tau}
\end{aligned}
$$

where $i=1$ and $2, \delta_{i}^{j}$ is the Kronecker delta, the admittance derivative functions have been defined in (2), and $\tau=d / c$ is the time delay. To analyze the stability of the steady-state solution $\left(V_{1}, V_{2}, \phi, \omega\right)$, a small perturbation will be introduced in system (23), which will give rise to increments in the time-varying phasors $X_{i}(t)=V_{i} e^{j \phi_{i}}+\delta X_{i}(t)$, where

$$
\begin{aligned}
\delta X_{i}(t) & =\int_{-\omega / 2}^{\omega / 2} \delta X_{i}(\Omega) e^{j \Omega t} d \Omega \\
& =\left(\delta V_{i}(t)+j V_{i} \delta \phi_{i}(t)\right) e^{j \phi_{i}}
\end{aligned}
$$

and $\delta V_{i}(t)$ and $\delta \phi_{i}(t)$ are the amplitude and phase perturbation components of the $i$ th oscillator, respectively. In the frequency domain, the functions $H_{i}$ in (22) can be approached by their first-order Taylor series expansions about the steady-state solution, and removing the steady-state terms, the following perturbed system is obtained:

$$
\begin{aligned}
& H_{1 V}\left(X_{1}, X_{2}, \omega+\Omega\right) \delta V_{1}(\Omega)+H_{1 \phi}\left(X_{1}, X_{2}, \omega+\Omega\right) \delta \phi_{1}(\Omega) \\
& \quad-C(\omega+\Omega) e^{-j(\omega+\Omega) \tau} \delta X_{2}(\Omega)=0 \\
& H_{2 V}\left(X_{1}, X_{2}, \omega+\Omega\right) \delta V_{2}(\Omega)+H_{2 \phi}\left(X_{1}, X_{2}, \omega+\Omega\right) \delta \phi_{2}(\Omega) \\
& \quad-C(\omega+\Omega) e^{-j(\omega+\Omega) \tau} \delta X_{1}(\Omega)=0
\end{aligned}
$$

where $H_{i V} \equiv \partial H_{i} / \partial V_{i}$ and $H_{i \phi} \equiv \partial H_{i} / \partial \phi_{i}$. Now, system (25) will be translated to the envelope domain by approaching $Y_{i}\left(V_{i}, \omega+\Omega\right) \simeq Y_{i}\left(V_{i}, \omega\right)+Y_{i \omega} \Omega$ and $C(\omega+\Omega) \simeq$ $C(\omega)+C_{\omega}(\omega) \Omega$ and applying the inverse Fourier transform

$$
\begin{aligned}
& Y_{1}^{0} \delta X_{1}(t)+Y_{1 V} X_{1} \delta V_{1}(t)-j Y_{1 \omega} \delta \dot{X}_{1}(t) \\
& \quad+Y_{c} \delta X_{2}(t-\tau)+j C_{\omega}(\omega) e^{-j \omega \tau} \delta \dot{X}_{2}(t-\tau)=0 \\
& Y_{2}^{0} \delta X_{2}(t)+Y_{2 V} X_{2} \delta V_{2}(t)-j Y_{2 \omega} \delta \dot{X}_{2}(t) \\
& \quad+Y_{c} \delta X_{1}(t-\tau)+j C_{\omega}(\omega) e^{-j \omega \tau} \delta \dot{X}_{1}(t-\tau)=0
\end{aligned}
$$

where $Y_{i}^{0} \equiv Y_{i}\left(V_{i}, \omega\right)$ and the time-delayed terms $\delta X_{i}(t-\tau)$ arise naturally when applying the inverse Fourier transform $\Im^{-1}\left\{e^{-j \Omega \tau} \delta X_{i}(\Omega)\right\}=\delta X_{i}(t-\tau)$. Now, to obtain the poles, linear system (26) is translated to the Laplace domain, which yields

$$
\begin{aligned}
\left(Y_{1}^{0}\right. & \left.+Y_{1 V} V_{1}-j Y_{1 \omega} s\right) \delta V_{1}+\left(Y_{1}^{0}-j Y_{1 \omega} s\right) j V_{1} \delta \phi_{1} \\
& +\left(Y_{c}+j C_{\omega}(\omega) e^{-j \omega \tau} s\right) e^{-s \tau}\left(\delta V_{2}+j V_{2} \delta \phi_{2}\right) e^{j \phi}=0 \\
\left(Y_{2}^{0}\right. & \left.+Y_{2 V} V_{2}-j Y_{2 \omega} s\right) e^{j \phi} \delta V_{2}+\left(Y_{2}^{0}-j Y_{2 \omega} s\right) j V_{2} e^{j \phi} \delta \phi_{2} \\
& +\left(Y_{c}+j C_{\omega}(\omega) e^{-j \omega \tau} s\right) e^{-s \tau}\left(\delta V_{1}+j V_{1} \delta \phi_{1}\right)=0 .
\end{aligned}
$$

Considering also the complex-conjugate equations of system (27), one obtains a complex system of the form $A(s) \delta \bar{P}(s)=\overline{0}$ where $\delta \bar{P} \equiv\left(\delta \phi_{1}, \delta \phi_{2}, \delta V_{1}, \delta V_{2}\right)^{T}$. Then, the system poles are the zeroes $s=\lambda$ of the characteristic determinant $\operatorname{det} A(s)$. Note that, from (27), the matrix $A(0)$ is given by

$$
A(0)=\left(\begin{array}{cc}
\frac{\partial \bar{H}}{\partial \bar{\phi}} & \frac{\partial \bar{H}}{\partial \bar{V}} \\
\left(\frac{\partial \bar{H}}{\partial \bar{\phi}}\right)^{*} & \left(\frac{\partial \bar{H}}{\partial \bar{V}}\right)^{*}
\end{array}\right)
$$

where $*$ means the complex conjugation, $\bar{H}=\left(H_{1} H_{2}\right)^{T}, \bar{\phi}=$ $\left(\phi_{1} \phi_{2}\right)^{T}$, and $\bar{V}=\left(V_{1} V_{2}\right)^{T}$. One can easily prove that the matrix $A(0)$ is singular since, due to the autonomy of the coupled system, the equations in (22) must be fulfilled for any constant phase shift $\alpha$, leading to $\phi_{1}=\alpha$ and $\phi_{2}=\phi+\alpha$. Therefore,

$$
\overline{0}=\frac{\partial \bar{H}}{\partial \alpha}=\frac{\partial \bar{H}}{\partial \bar{\phi}} \frac{\partial \bar{\phi}}{\partial \alpha}, \quad \frac{\partial \bar{\phi}}{\partial \alpha}=\left(\begin{array}{l}
1 \\
1
\end{array}\right) .
$$

Combining (28) and (29), one can easily verify that the matrix $A(0)$ has the eigenvector $\left(\begin{array}{llll}1 & 1 & 0 & 0\end{array}\right)^{T}$, associated with the eigenvalue 0 , so one of the zeroes of $\operatorname{det} A(s)$ is $\lambda=0$. Because this root exists for all the parameter values, it will be convenient to remove it from the characteristic determinant, which can be done by considering the function $p(s)=$ $\operatorname{det} A(s) / s$. Note that considering the delay effects on the signal envelopes in system (26) provides a deep insight into the coupled system stability. Had these delays been approached by a first-order Taylor expansion in $\tau$, the resulting system would have been of fourth order, yielding four poles, unlike the infinite-dimension system (27). The dominant poles of the delayed system (27) can be obtained by applying a pole-zero identification technique to the complex function $1 / p(j \Omega)$, with $\Omega \in(0, \omega / 2]$.

The analysis described has been applied to the closed solution curves of Fig. 4(b), traced versus $V_{\text {dc }}$. Fig. 8 shows the variation of the real part of the dominant poles versus $V_{\mathrm{dc}}$ and shows that, at each turning point, a real pole passes through zero. The upper section of the curve corresponds to unstable solutions (poles in red) and the lower section to stable solutions (poles in blue). We have also analyzed the stability of the two solutions in Fig. 5, obtained for $G=2 \mathrm{~dB}$ versus the distance $d$. The variation of their corresponding dominant poles is shown in Fig. 9. The lower amplitude curve [Fig. 9(a)] is stable, whereas the higher amplitude one [Fig. 9(b)] is unstable. As gathered from the evolution of the dominant poles of the stable solution in Fig. 9(a), the reduction of the stability margin as $d$ increases is very slow. In fact, this reduction is not noticeable in the distance range considered in Fig. 9. 


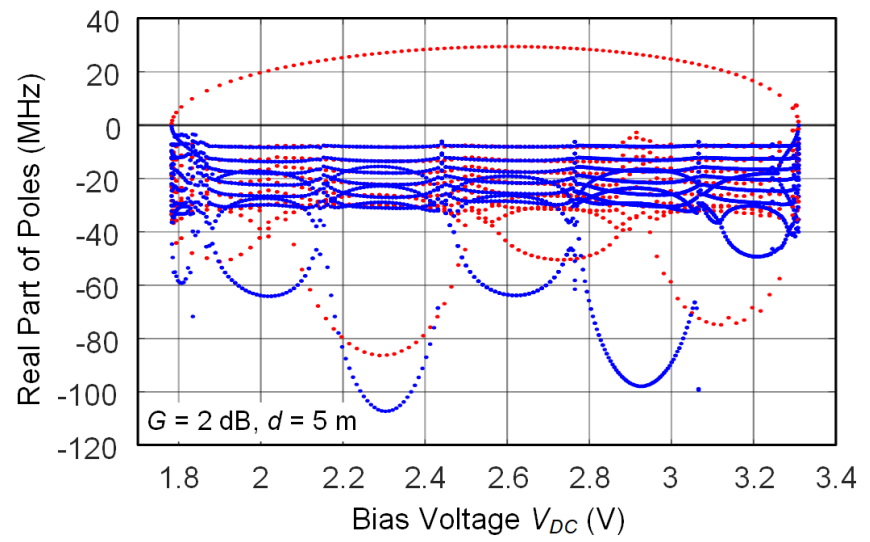

Fig. 8. Variation of the real part of the dominant poles of the closed curves in Fig. 4 (traced versus $V_{\mathrm{dc}}$ ). Note the large number of detected poles, resulting from the consideration of delay effects in the signal envelopes. The upper section of the curve corresponds to unstable solutions (poles in red) and the lower section the curve corresponds to stable solutions (poles in blue). A real pole passes through zero at each of the two turning points from which the system becomes unlocked.

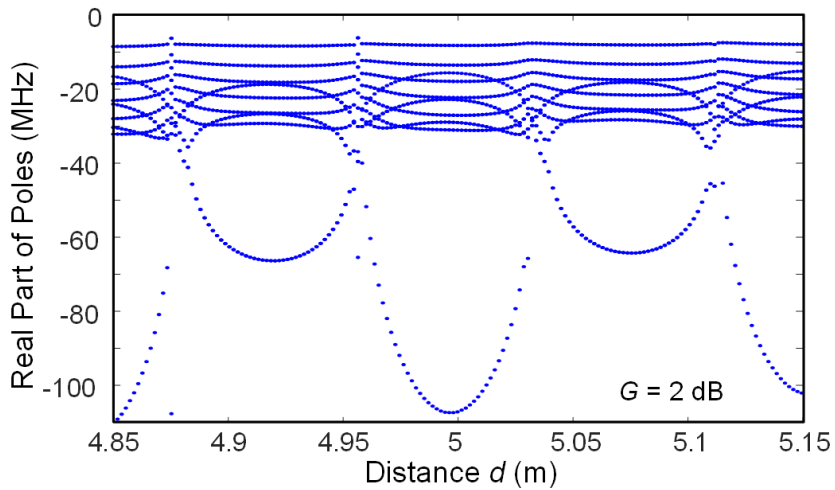

(a)

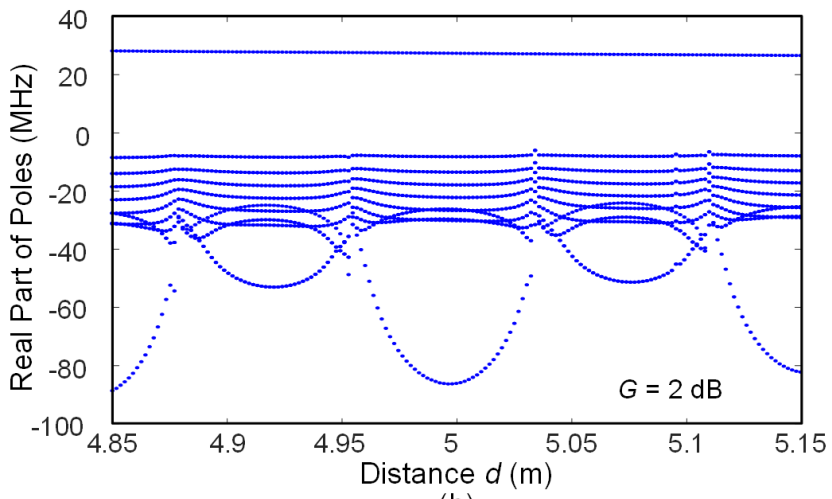

(b)

Fig. 9. Stability analysis of the solution curves in Fig. 5. The real part of the dominant poles has been traced versus the distance $d$. (a) Lower amplitude solution, with stable behavior. (b) Higher amplitude solution, with unstable behavior.

\section{Analysis Under Modulated Conditions}

In the presence of a modulation signal $V_{\mathrm{dc}}(t)$ in the bias voltage of the first oscillator, the phasors $X_{1}$ and $X_{2}$ will become time-varying. Departing from system (22) and considering that the complex-frequency increment $j \omega$ acts like a time differentiator, one obtains the following system, which
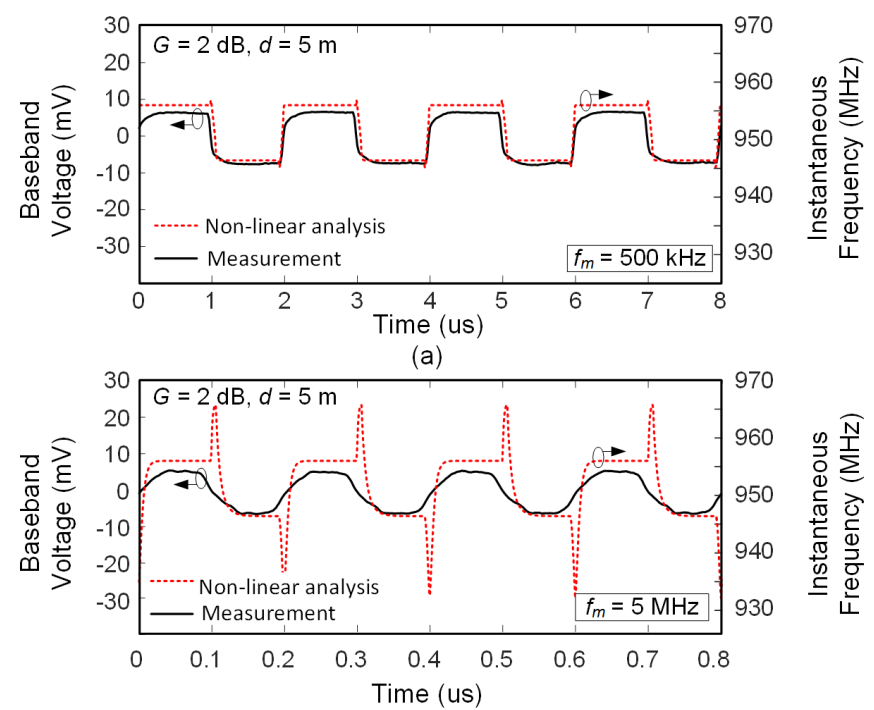

(b)

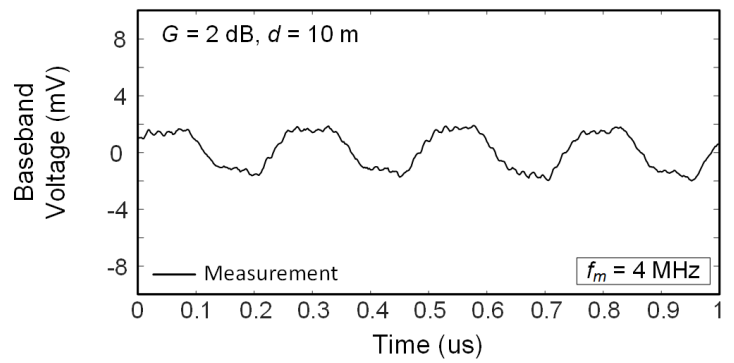

(c)

Fig. 10. Operation under frequency modulation. A rectangular signal is introduced in the bias voltage of the first oscillator. This voltage signal has an excursion $V_{\mathrm{pp}}=0.5 \mathrm{~V}$ about the offset $V_{\text {offset }}=2.3 \mathrm{~V}$. (a) Analysis and measurements for $d=5 \mathrm{~m}$ and $f_{m}=500 \mathrm{kHz}$. (b) Analysis and measurements for $d=5 \mathrm{~m}$ and $f_{m}=5 \mathrm{MHz}$. (c) Measurements for $d=10 \mathrm{~m}$ and $f_{m}=4 \mathrm{MHz}$

governs the dynamics of the complex envelopes $X_{1}(t)$, and $X_{2}(t)$ :

$$
\begin{aligned}
& Y_{1}(t) X_{1}(t)-j Y_{1 \omega} \dot{X}_{1}(t)+Y_{c} X_{2}(t)-j Y_{c \omega} \dot{X}_{2}(t)=0 \\
& Y_{2}(t) X_{2}(t)-j Y_{2 \omega} \dot{X}_{2}(t)+Y_{c} X_{1}(t)-j Y_{c \omega} \dot{X}_{1}(t)=0
\end{aligned}
$$

where

$$
\begin{aligned}
Y_{i}(t)= & Y_{i V}\left(V_{i}(t)-V_{o i}\right)+Y_{i \omega}\left(\omega-\omega_{o i}\right) \\
& +\delta_{i}^{1} Y_{\mathrm{DC}}\left(V_{\mathrm{dc}}(t)-V_{\mathrm{dco}}\right) \\
Y_{c \omega}= & -\left(C_{\omega}(\omega)-j \tau C(\omega)\right) e^{-j \omega \tau}
\end{aligned}
$$

and $\omega$ is the frequency of the steady-state solution.

System (30) will be used to obtain the system response to a rectangular signal introduced in the bias voltage of the first oscillator. This voltage signal has an excursion $V_{\mathrm{pp}}=0.5 \mathrm{~V}$ about the offset $V_{\text {offset }}=2.3 \mathrm{~V}$ at the modulation frequency $f_{m}$, and the analysis will be carried out for a gain $G=2 \mathrm{~dB}$ and distance $d=5 \mathrm{~m}$ between the oscillators. Fig. 10(a), corresponding to $f_{m}=500 \mathrm{kHz}$, presents the modulated frequency predicted with (30) and the measured demodulated signal in the bias circuitry of the second oscillator. Fig. 10(b) performs the same comparison for $f_{m}=5 \mathrm{MHz}$ at the limit 


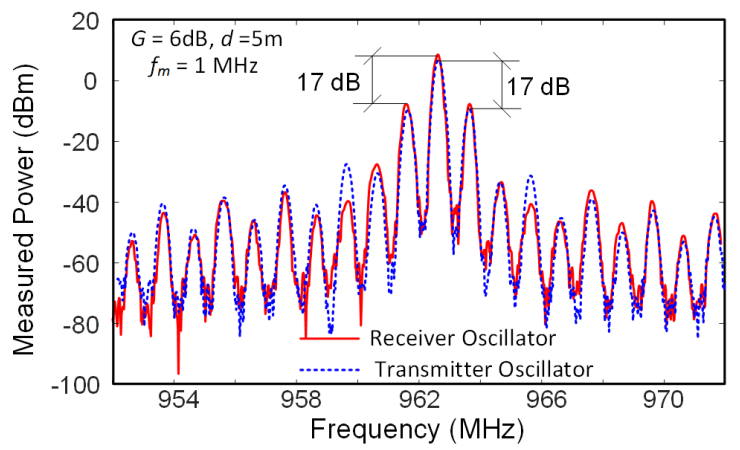

(a)

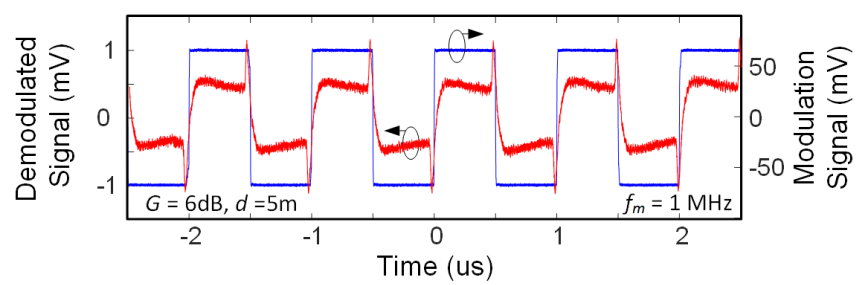

(b)

Fig. 11. Demodulation of a rectangular signal at $1 \mathrm{MHz}$. (a) Spectra of the transmitter and receiver oscillator. (b) Modulation and demodulated signals.

of the demodulation capabilities. In the analysis through (30), the rectangular signal is modeled with a piecewise function, so the derivative discontinuities give rise to the peaks observed in the instantaneous frequency. Fig. 10(c) shows the measured demodulated signal at twice the distance $d=10 \mathrm{~m}$ when using $f_{m}=4 \mathrm{MHz}$. Note that the aim of these tests has been to determine the system operation limits.

To fully characterize the system operation, we have performed some additional measurements. In Fig. 11(a), we compare the spectra of the transmitter and receiver oscillator under a rectangular modulation signal of $1 \mathrm{MHz}$. There is a higher power at the transmitter oscillator since its spectrum is measured at the output port, whereas that of the receiver oscillator is measured at the input port. Except for the different levels, the spectra are nearly identical even though they have been obtained in two sequential measurements, after disconnecting the spectrum analyzer from the first oscillator and connecting it to the second one. The modulation and demodulated signals are shown in Fig. 11(b).

Due to the minimum-amplitude limitations of the arbitrary waveform generator, we have estimated the nonlinearity of the Tx/Rx system by measuring the variation of the output $\mathrm{dc}$ voltage of the receiver oscillator versus the bias voltage of the transmitter one. The results are shown in Fig. 12(a). For $V_{\mathrm{dc}}$ lower than $1.7 \mathrm{~V}$, the system is unlocked. For $V_{\mathrm{dc}}$ higher than $3 \mathrm{~V}$, there is a saturation effect. We have also measured the voltage gain from the baseband input of the modulator oscillator to the baseband output of the receiver one. This has been done by introducing a sinusoidal tone at the frequency $f_{m}$, with the system operating in a linear region. When sweeping $f_{m}$, one obtains the results in Fig. 12(b). The loss is due to the limitations in the simple frequency demodulation mechanism used, based on the detection of

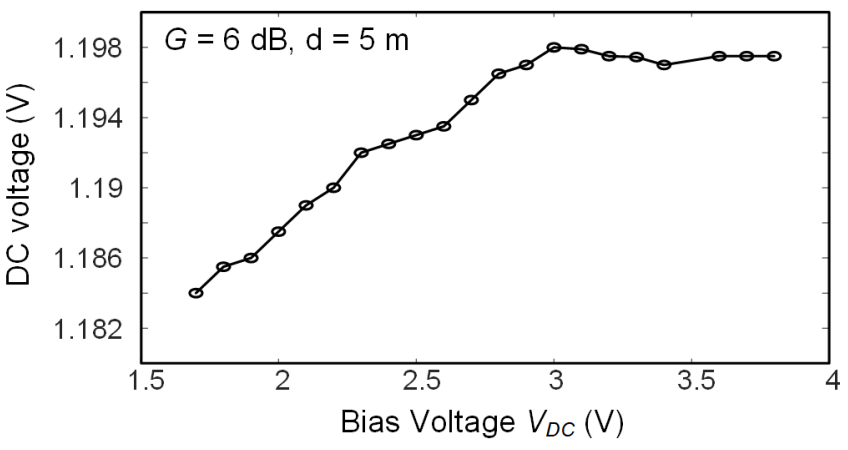

(a)

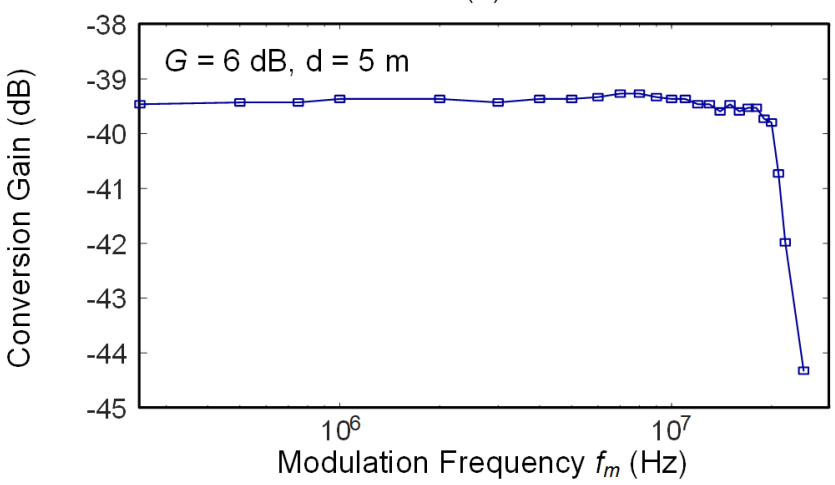

(b)

Fig. 12. Experimental response. (a) Variation of the dc output voltage in the receiver oscillator versus the bias voltage $V_{\mathrm{dc}}$ of the transmitter oscillator. (b) Measurement of the voltage gain from the baseband input in the modulator oscillator to the baseband output in the demodulator one. This measurement has been carried out by introducing a sinusoidal tone at the frequency $f_{m}$, with the system operating in a linear region.

the voltage drop in a resistor of the bias circuitry. Higher gain would be obtained with the aid of a baseband amplifier, as done in [4] and [17], which, for compactness, has not been introduced here.

\section{PhASE-NOISE ANALYSIS}

In the work [8], we addressed the noise analysis of a single Zero-IF SOM under a modulated injection-locked tone, with a low phase-noise spectral density. In the system considered here, the oscillation is self-generated, and its phase noise may significantly affect the demodulated signal, so the phasenoise analysis of the mutually locked oscillation is essential. To get insight into the influence of the phase noise, we will express the baseband term as $\Delta V_{\mathrm{dc}} \rightarrow \Delta V_{\mathrm{dc}}+\delta V_{\mathrm{dc}}(t)$, so the modulation signal $\delta V_{\mathrm{dc}}(t)$ is small enough for the coupled system to behave linearly about the nonmodulated steady state at $\Delta V_{\mathrm{dc}}$. To analyze the system behavior in the presence of noise perturbations, an equivalent noise current source $i_{i}(t)=$ $2 \operatorname{Re} I_{i}(t) e^{j \omega t}$, where $i=1$ and 2 , will be connected in parallel at the antenna node of each oscillator. These equivalent current sources are calculated by fitting the phase-noise spectrum of each oscillator in standalone operation, as described in [10]. Each phasor $I_{i}(t)=I_{i}^{w}(t)+I_{i}^{f}(t)$ is constituted by the contributions of the white and upconverted flicker noise. The phasors $I_{i}(t)$ are stochastic processes that, in the frequency 
domain, fulfill the following correlation properties:

$$
\begin{aligned}
\left\langle I_{i}^{w}(\Omega) I_{j}^{w}(\Omega)^{*}\right\rangle & =\delta_{i}^{j} \Gamma_{i} \\
\left\langle I_{i}^{f}(\Omega) I_{j}^{f}(\Omega)^{*}\right\rangle & =\delta_{i}^{j} \frac{k_{f, i}}{\Omega} .
\end{aligned}
$$

The dynamics of the resulting system can be analyzed by introducing the modulation component $\delta V_{\mathrm{dc}}(t)$ in (27), which yields

$$
\begin{gathered}
\left(Y_{1}^{0}+Y_{1 V} V_{1}-j Y_{1 \omega} s\right) \delta V_{1}+\left(Y_{1}^{0}-j Y_{1 \omega} s\right) j V_{1} \delta \phi_{1}+Y_{\mathrm{DC}} \delta V_{\mathrm{dc}} \\
+\left(Y_{c}+j C_{\omega}(\omega) e^{-j \omega \tau} s\right) e^{-s \tau}\left(\delta V_{2}+j V_{2} \delta \phi_{2}\right) e^{j \phi}=I_{1} \\
\left(Y_{2}^{0}+Y_{2 V} V_{2}-j Y_{2 \omega} s\right) e^{j \phi} \delta V_{2}+\left(Y_{2}^{0}-j Y_{2 \omega} s\right) j V_{2} e^{j \phi} \delta \phi_{2} \\
+\left(Y_{c}+j C_{\omega}(\omega) e^{-j \omega \tau} s\right) e^{-s \tau}\left(\delta V_{1}+j V_{1} \delta \phi_{1}\right)=I_{2} .
\end{gathered}
$$

Equation (33) shows that the instantaneous frequency $\delta \dot{\phi}_{2}(t)$ of the receiver oscillator is modulated both by the equivalent noise sources and the baseband modulation signal $\delta V_{\mathrm{dc}}(t)$ of the transmitter oscillator. This perturbation can be expressed as

$$
\delta \omega_{2}(s)=s \delta \phi_{2}(s)=s \delta \phi_{2, n}(s)+a_{0}(s) \delta V_{\mathrm{dc}}(s)
$$

where $\delta \phi_{2, n}(s)$ is the phase noise of the receiver oscillator, which is given by

$$
\delta \phi_{2, n}(s)=a_{1}(s) I_{1}(s)+a_{2}(s) I_{2}(s)
$$

where the transfer functions $a_{i}(s)$ in (34) and (35) can be derived from the linear system (33). Then, as described in [8], the demodulation mechanism will downconvert the spectral components of the frequency perturbation $\delta \omega_{2}(t)$ into the baseband component of the receiver oscillator. Equation (34) shows that these components are produced by both the receiver phase noise and the transmitter modulation signal. In the following, a mathematical formulation to calculate the phasenoise spectral density is provided.

In the absence of modulation $\left(\delta V_{\mathrm{dc}}(t)=0\right)$, system (33) can be expressed in the frequency domain as

$$
\begin{aligned}
A(j \Omega) \delta \bar{P}(\Omega) & =\bar{I}(\Omega) \\
\bar{I} & =\left(\begin{array}{llll}
I_{1} & I_{1}^{*} & I_{2} & I_{2}^{*}
\end{array}\right)^{T}
\end{aligned}
$$

where the matrix $A(s)$ was introduced in (27). Then, one should obtain the correlation matrix of the perturbation components

$$
\begin{aligned}
\left\langle\delta \bar{P}(\Omega) \delta \bar{P}(\Omega)^{+}\right\rangle & =\frac{B(j \Omega) C(\Omega) B(j \Omega)^{+}}{\Omega^{2}|p(j \Omega)|^{2}} \\
C(\Omega) & =\left(\begin{array}{cccc}
R_{1}(\Omega) & 0 & 0 & 0 \\
0 & R_{1}(\Omega) & 0 & 0 \\
0 & 0 & R_{2}(\Omega) & 0 \\
0 & 0 & 0 & R_{2}(\Omega)
\end{array}\right) \\
R_{i}(\Omega) & =\Gamma_{i}+\frac{k_{f, i}}{\Omega}
\end{aligned}
$$

where $B(j \Omega)$ is the adjugate matrix of $A(j \Omega)$ and $C(\Omega)$ is a diagonal matrix containing the correlation components $R_{i}(\Omega)$ of the noise sources in each $i$ th oscillator. The power spectral density of the perturbation components is then given by

$$
\begin{aligned}
\left\langle\left|\delta P_{k}(\Omega)\right|^{2}\right\rangle & =\frac{1}{\Omega^{2}} \frac{\sum_{p=1}^{4}\left|b_{k p}(\Omega)\right|^{2} C_{\mathrm{pp}}(\Omega)}{|p(j \Omega)|^{2}} \\
& =c_{k 1}(\Omega) R_{1}(\Omega)+c_{k 2}(\Omega) R_{2}(\Omega)
\end{aligned}
$$

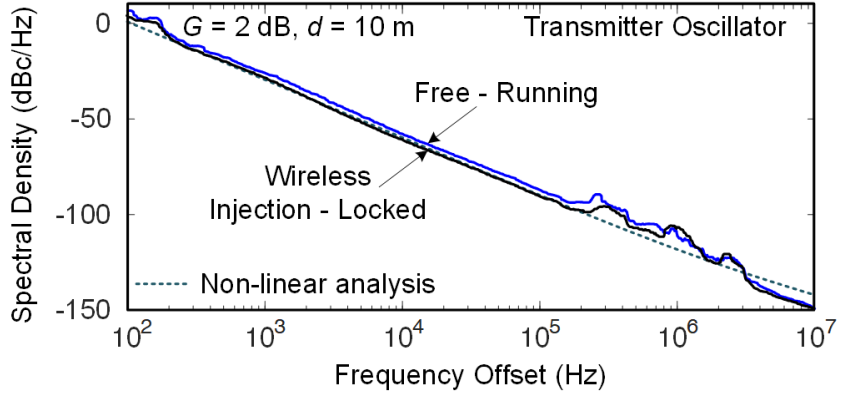

(a)

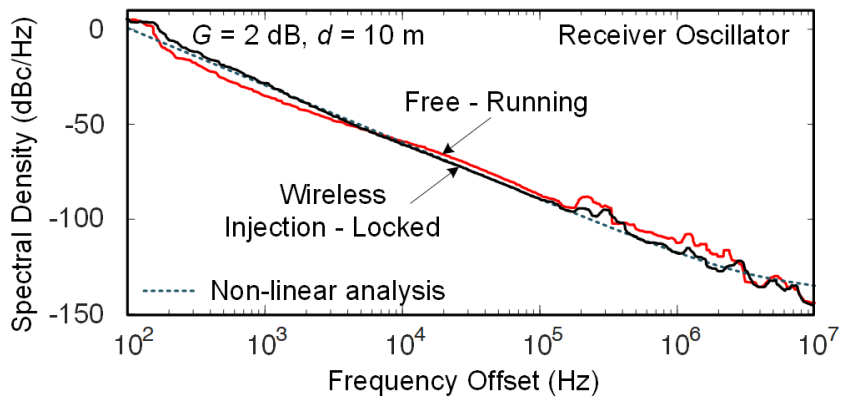

(b)

Fig. 13. Phase-noise spectral density of the system in Fig. 1 with antenna gain $G=2 \mathrm{~dB}$ and distance $d=10 \mathrm{~m}$. The results provided by (38) are compared with those obtained in uncoupled operation and with measurements carried out with the R\&S FSWP8 - phase-noise analyzer. (a) Transmitter oscillator. (b) Receiver oscillator.

where $k=1, \ldots, 4, b_{k p}(\Omega)(p=1, \ldots, 4)$ are the components of the $i$ th row of the adjugate matrix $B(j \Omega)$ and $c_{k 1}(\Omega)$ and $c_{k 2}(\Omega)$ are the noise coefficients of the $k$ th perturbation variable. Note that the structure of system (27) assures that both $\left|b_{k p}(0)\right|^{2}$ and $|p(0)|^{2}$ are bounded. In the case of the phase-noise perturbations ( $k=1$ and 2$),\left|b_{k p}(0)\right|^{2}$ is, in general, bigger than zero. Then, (37) shows that, provided that $p(0) \neq 0$, the perturbation system (27) contains only one pole at zero, so, for small values of $\Omega$, the phase-noise coefficients behave as $c_{k i}(\Omega) \sim 1 / \Omega^{2}$ for $k=1$ and 2 . Then, the near carrier phase-noise spectrum shows the typical $-30 \mathrm{~dB} /$ decade slope followed by a $-20-\mathrm{dB} /$ decade slope produced by the flicker and white noise sources, respectively. In the case of the amplitude noise components, the inspection of matrix $A(0)$ in expression (28) provides $\left|b_{k p}(0)\right|^{2}=0$ for $k=3$ and 4 . Then, the numerator has in general the form $\left|b_{k p}(\Omega)\right|^{2}=O\left(\Omega^{2}\right)$, canceling the $1 / \Omega^{2}$ behavior.

The analysis based on (38) has been applied to the system in Fig. 1 with antenna gain $G=2 \mathrm{~dB}$ and distance $d=$ $10 \mathrm{~m}$. The phase-noise spectra in the transmitter and receiver oscillators are shown in Fig. 13. In the two cases, the results are compared with those obtained in uncoupled operation and with measurements carried out with the R\&S FSWP8 phasenoise analyzer. The phase noise of each oscillator is measured at the port that is not connected to the antenna using an R\&S FSWP8 phase-noise analyzer. Note that the timing noise, which is the most relevant contribution, does not depend on the observation node [29]. The phase noise is rather small in the two cases, in consistency with the good demodulation capabilities of the system, shown in Section VI. The phase noise in wireless-locked operation is about $3 \mathrm{~dB}$ lower than 
that in standalone operation. The reduction obtained is the one expected in a coupled system of identical oscillators, that is, $10 \log N$, where $N$ is the number of oscillator elements [30].

\section{CONCLUSION}

An in-depth investigation of a wireless communication link established between two Zero-IF SOMs has been presented for possible application in systems requiring compact size, as well as low cost and power consumption. Two types of formulation based on realistic oscillator models extracted from HB simulations have been derived: one is nonlinear in the voltage amplitudes and solved with arc-length continuation and the other is linear in these amplitudes. The latter is less accurate but provides insight into the main properties of the locked-system behavior. Systems based on single Zero-IF SOM injection locked by a signal undergoing propagation effects can be analyzed with these formulations as a particular case. With two identical Zero-IF SOMs, an optimum performance is achieved when there is a high ratio between the amplitudes of the oscillator acting as a transmitter and the one acting as a receiver at the antenna connection nodes. The study includes the stability and phase noise analysis of the coupled system solution. Good performance under modulation by a rectangular signal of $5 \mathrm{MHz}$ has been achieved up to $10 \mathrm{~m}$, as has been experimentally validated. In view of these results, we believe that the inclusion of two antennas in each Zero-IF SOM could enable bidirectional communications, though the evaluation of such system is out of the scope of this work.

\section{REFERENCES}

[1] P. Burasa, N. G. Constantin, and K. Wu, "Low-power injection-locked Zero-IF self-oscillating mixer for high Gbit/s data-rate battery-free active $\mu$ RFID tag at millimeter-wave frequencies in 65-nm CMOS," IEEE Trans. Microw. Theory Techn., vol. 64, no. 4, pp. 1055-1065, Apr. 2016.

[2] P. Burasa, N. Constantin, and K. Wu, "Low-power injection-locked zero-IF self-oscillating mixer for self-powered millimeter-wave identification (MMID) active tag in 65-nm CMOS," in Proc. IEEE Radio Freq. Integr. Circuits Symp. (RFIC), May 2015, pp. 259-262.

[3] H. Shan, J. Peterson III, S. Hathorn, and S. Mohammadi, "The RFID connection: RFID technology for sensing and the Internet of Things," IEEE Microw. Mag., vol. 19, no. 7, pp. 63-79, Nov./Dec. 2018.

[4] P. Burasa, B. Mnasri, and K. Wu, "Millimeter-wave CMOS sourceless receiver architecture for $5 \mathrm{G}$-served ultra-low-power sensing and communication systems," IEEE Trans. Microw. Theory Techn., vol. 67, no. 5, pp. 1688-1696, May 2019.

[5] M. Ponton, A. Herrera, and A. Suarez, "Analysis and design of a concurrent dual-band self-oscillating mixer," in IEEE MTT-S Int. Microw. Symp. Dig., Los Angeles, CA, USA, Aug. 2020, pp. 4-6.

[6] M. Ponton, A. Herrera, and A. Suarez, "Double functionality concurrent dual-band self-oscillating mixer," IEEE Trans. Microw. Theory Techn., vol. 69, no. 1, pp. 786-802, Jan. 2021.

[7] M. Sagawa, M. Makimoto, and S. Yamashita, "Geometrical structures and fundamental characteristics of microwave stepped-impedance resonators," IEEE Trans. Microw. Theory Techn., vol. 45, no. 7, pp. 1078-1085, Jul. 1997.

[8] M. Ponton, S. Sancho, A. Herrera, and A. Suarez, "Analysis of noise and dynamical effects in zero-IF self-oscillating mixers," in IEEE MTT-S Int. Microw. Symp. Dig., Atlanta, GA, USA, Jun. 2021, pp. 180-183.

[9] N. B. Buchanan and V. Fusco, "Single VCO chipless RFID near-field reader," Electron. Lett., vol. 52, no. 23, pp. 1958-1960, 2016.

[10] F. Ramírez, M. Pontón, S. Sancho, and A. Suárez, "Phase-noise analysis of injection-locked oscillators and analog frequency dividers," IEEE Trans. Microw. Theory Techn., vol. 56, no. 2, pp. 393-407, Feb. 2008.

[11] N. B. Buchanan and V. Fusco, "Simplified, high performance transceiver for phase modulated RFID applications," in Proc. Eur. Microw. Conf. (EuMC), Paris, France, Sep. 2015, pp. 104-106.
[12] S.-E. Chen and K.-W. Cheng, "A $433 \mathrm{MHz} 54 \mu \mathrm{W}$ OOK/FSK/PSK compatible wake-up receiver with $11 \mu \mathrm{W}$ low-power mode based on injection-locked oscillator," in Proc. 42nd Eur. Solid-State Circuits Conf. (ESSCIRC), Lausanne, Switzerland, Sep. 2016, pp. 137-140.

[13] F. Amato and S. Hemour, "The harmonic tunneling tag: A dual-band approach to backscattering communications," in Proc. IEEE Int. Conf. RFID Technol. Appl. (RFID-TA), Pisa, Italy, Sep. 2019, pp. 244-247.

[14] F. Amato, C. W. Peterson, B. P. Degnan, and G. D. Durgin, "Tunneling RFID tags for long-range and low-power microwave applications," IEEE J. Radio Freq. Identif., vol. 2, no. 2, pp. 93-103, Jun. 2018.

[15] B. Razavi, "A study of injection locking and pulling in oscillators," IEEE J. Solid-State Circuits, vol. 39, no. 9, pp. 1415-1424, Sep. 2004.

[16] R. Adler, "A study of locking phenomena in oscillators," Proc. IRE, vol. 34, no. 6, pp. 351-357, Jun. 1946.

[17] P. Burasa, B. Mnasri, and K. Wu, "A sourceless low-power mmW receiver architecture using self-oscillating mixer array in 65-nm CMOS," in Proc. IEEE Wireless Power Transf. Conf. (WPTC), Montreal, QC, Canada, Jun. 2018, pp. 1-3.

[18] A. Suárez, F. Ramírez, and S. Sancho, "Stability and noise analysis of coupled-oscillator systems," IEEE Trans. Microw. Theory Techn., vol. 59, no. 4, pp. 1032-1046, Apr. 2011.

[19] M. Pontón and A. Suárez, "Wireless injection locking of oscillator circuits," IEEE Trans. Microw. Theory Techn., vol. 64, no. 12, pp. 4646-4659, Dec. 2016.

[20] M. Pontón and A. Suárez, "Oscillation modes in symmetrical wirelesslocked systems," IEEE Trans. Microw. Theory Techn., vol. 66, no. 5, pp. 2495-2510, May 2018.

[21] M. Pontón, A. Herrera, and A. Suárez, "Wireless-coupled oscillator systems with an injection-locking signal," IEEE Trans. Microw. Theory Techn., vol. 67, no. 2, pp. 642-658, Feb. 2019.

[22] F. Ramírez, S. Sancho, M. Pontón, and A. Suárez, "Two-scale envelopedomain analysis of injected chirped oscillators," IEEE Trans. Microw. Theory Techn., vol. 66, no. 12, pp. 5449-5461, Dec. 2018.

[23] F. Ramírez, M. E. de Cos, and A. Suárez, "Nonlinear analysis tools for the optimized design of harmonic-injection dividers," IEEE Trans. Microw. Theory Techn., vol. 51, no. 6, pp. 1752-1762, Jun. 2003.

[24] J. M. T. Thomson and H. B. Stewart, Nonlinear Dynamics and Chaos. Hoboken, NJ, USA: Wiley, 2002

[25] J. Griffiths, Radio Wave Propagation and Antennas: An Introduction. Upper Saddle River, NJ, USA: Prentice-Hall, 1986.

[26] A. Suárez, Analysis and Design of Autonomous Microwave Circuits. Hoboken, NJ, USA: Wiley, 2009.

[27] H. B. Keller, "Numerical solution of bifurcation and nonlinear eigenvalue problems," in Applications of Bifurcation Theory, P. Rabinowitz, Ed. New York, NY, USA: Academic, 1977, pp. 359-384.

[28] J. S. Roychowdhury and R. C. Melville, "Homotopy techniques for obtaining a DC solution of large-scale MOS circuits," in Proc. 33rd Design Automat. Conf., 1996, pp. 286-291.

[29] S. Sancho, A. Suárez, J. Dominguez, and F. Ramírez, "Analysis of nearcarrier phase-noise spectrum in free-running oscillators in the presence of white and colored noise sources," IEEE Trans. Microw. Theory Techn., vol. 58, no. 3, pp. 587-601, Mar. 2010.

[30] H.-C. Chang, X. Cao, U. K. Mishra, and R. A. York, "Phase noise in coupled oscillators: Theory and experiment," IEEE Trans. Microw. Theory Techn., vol. 45, no. 5, pp. 604-615, May 1997.

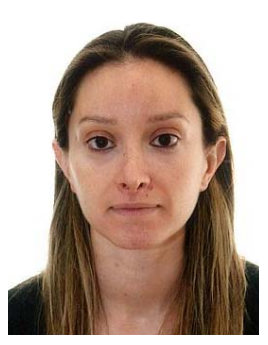

Mabel Pontón (Member, IEEE) was born in Santander, Spain. She received the bachelor's degree in telecommunication engineering, the master's degree in information technologies and wireless communications systems, and the Ph.D. degree from the University of Cantabria, Santander, in 2004, 2008, and 2010, respectively.

In 2006, she joined the Communications Engineering Department, University of Cantabria. From 2011 to 2013, she was with the Group of Electronic Design and Applications, Georgia Institute of Technology, Atlanta, GA, USA, as a Post-Doctoral Research Fellow. Her current research interests include the nonlinear analysis and simulation of radio frequency and microwave circuits, with an emphasis on phase noise, stability, and bifurcation analysis of complex oscillator topologies. 


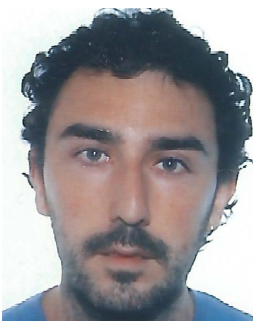

Sergio Sancho (Member, IEEE) received the Licentiate degree in electronic physics from Basque Country University, Basque Country, Leioa, Spain, in 1997 and the Ph.D. degree in electronic engineering from the Communications Engineering Department, University of Cantabria, Santander, Spain, in February 2002

In 1998, he joined the Communications Engineering Department, University of Cantabria, where he currently works as an Associate Professor. His research interests include the nonlinear analysis of microwave autonomous circuits and frequency synthesizers, including stochastic and phase-noise analysis.

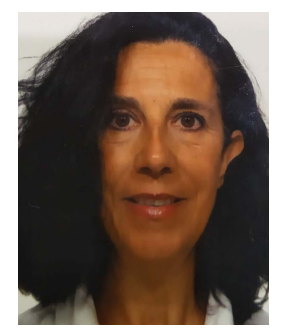

Amparo Herrera was born in Asturias, Spain, in 1963. She received the Licentiate degree in electronic physics from the University of Cantabria, Santander, Spain, in June 1987, and the Ph.D. degree in electronics from the University of Cantabria in June 1995.

In 1987, she joined the Centro de Investigación y Desarrollo de la Armada (CIDA) (Research and Development Spanish Army Centre), Madrid, Spain, and the Research and Development Spanish Army Centre, working on developing the RF and Microwave Laboratory up to 1990 when she joined the Department of Electronics, University of Cantabria, working on monolithic microwave integrated circuit (MMIC) design. From 1992 to 1995, she collaborated with the Laboratory Philips Microwave Limeil (actually OMMIC), Limeil, France; as a result of this collaboration, she presents her Ph.D. thesis. In this work, she had designed a high-efficiency power amplifier for digital European cordless telecommunications (DECT) and digital communication systems (DCS) applications a work for the Philips Microwave Limeill foundry (now called OMMIC). Since 1995, she has been an Associate Professor and a member of the Communications Engineering Department, University of Cantabria. She has taken part as a main researcher in a big number of Spanish and European projects of the III, IV, V, and VI frameworks and the Spanish National Research and Development Plan. She has participated in a number of industrial projects with European and Spanish industries. She is currently working in the design and development of MMIC devices on Si-Ge and GaN pseudomorphic high-electron-mobility transistor (PHEMT) technologies. Her areas of interest include the MMIC and hybrid design of microwave circuits and, specially, the power and low-noise amplifier design and characterization.

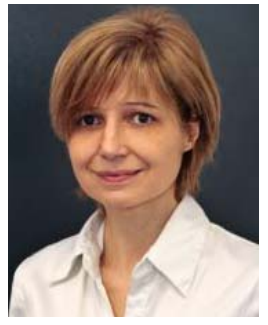

Almudena Suárez (Fellow, IEEE) was born in Santander, Spain. She received the Licentiate degree in electronic physics and the Ph.D. degree from the University of Cantabria, Santander, Spain, in 1987 and 1992, respectively, and the Ph.D. degree in electronics from the University of Limoges, Limoges, France, in 1993.

She is currently a Full Professor at the University of Cantabria and the Head of the Microwave Engineering and Radiocommunication Systems Research Group. She has authored the book Analysis and Design of Autonomous Microwave Circuits (IEEE-Wiley, 2009) and coauthored the book Stability Analysis of Nonlinear Microwave Circuit (Artech House, 2003).

Dr. Suárez is a member of the Technical Committee of IEEE International Microwave Symposium (IEEE MTT-S) and the European Microwave Week. She is also a member of the Board of Directors of the European Microwave Association. She was the Coordinator of the Communications and Electronic Technology Area for the Spanish National Evaluation and Foresight Agency (ANEP) from 2009 to 2013. She was the Chair of the 2014 and 2015 Editions of IEEE Topical Conference on RF/Microwave Power Amplifiers (PAWR), Newport Beach and San Diego. She was the General TPC Chair of European Microwave Week 2018. She was the Chair of the IEEE Subcommittee for the Best Paper Award at IEEE Microwave Magazine from 2017 to 2020. She was the Editor-in-Chief of the International Journal of Microwave and Wireless Technologies (Cambridge University Press journals) from 2013 to 2018. She is also an Associate Editor of IEEE Microwave Magazine and the IEEE TRANSACTIONS ON MiCROWAVE THEORY AND TeChNiques. She was an IEEE Distinguished Microwave Lecturer from 2006 to 2008 